BNL, -52519

\title{
DYNAMIC RESPONSE OF FLEXIBLE RETAINING WALLS
}

A. H. Younan, A. S. Veletsos and K. Bandyopadhyay

\section{bnl \\ aui}

January 1997

DEPARTMENT OF ADVANCED TECHNOLOGY

BROOKHAVEN NATIONAL LABORATORY, ASSOCIATED UNIVERSITIES, INC.

P.O. BOX 5000, UPTON, NEW YORK 11973-5000

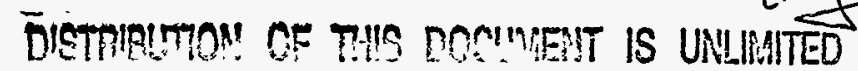

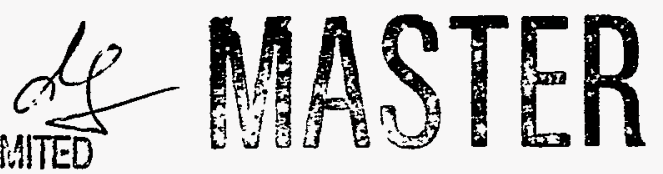




\section{DISCLAIMER}

This report was prepared as an account of work sponsored by an agency of the United States Government. Neither the United States Government nor any agency thereof, nor any of their employees, nor any of their contractors, subcontractors, or their employees, makes any warranty, express or implied, or assumes any legal liability or responsibility for the accuracy, completeness, or usefulness of any information, apparatus, product, or process disclosed, or represents that its use would not infringe privately owned rights. Reference herein to any specific commercial product, process, or service by trade name, trademark, manufacturer, or otherwise, does not necessarily constitute or imply its endorsement, recommendation, or favoring by the United States Government or any agency, contractor or subcontractor thereof. The views and opinions of authors expressed herein do not necessarily state or reflect those of the United States Government or any agency, contractor or subcontractor thereof. 
BNL- 52519

UC- 406

\title{
DYNAMIC RESPONSE OF FLEXIBLE RETAINING WALLS
}

\author{
A. H. Younan, A. S. Veletsos and K. Bandyopadhyay
}

\section{DISCLAIMER}

\begin{abstract}
This report was prepared as an account of work sponsored by an agency of the United States Government. Neither the United States Government nor any agency thereof, nor any of their employees, makes any warranty, express or implied, or assumes any legal liability or responsibility for the accuracy, completeness, or usefulness of any information, apparatus, product, or process disclosed, or represents that its use would not infringe privately owned rights. Reference herein to any specific commercial product, process, or service by trade name, trademark, manufacturer, or otherwise does not necessarily constitute or imply its endorsement, recommendation, or favoring by the United States Government or any agency thereof. The views and opinions of authors expressed herein do not necessarily state or reflect those of the United States Government or any agency thereof.
\end{abstract}

\section{January 1997}




\section{DISCLAIMER}

Portions of this document may be illegible in electronic image products. Images are produced from the best available original document. 


\begin{abstract}
Making use of an extension of a recently proposed, relatively simple, approximate method of analysis, a critical evaluation is made of the response to horizontal ground shaking of flexible walls retaining a uniform, linear, viscoelastic stratum of constant thickness and semiinfinite extent in the horizontal direction. Both cantilever and top-supported walls are examined. Following a detailed description of the method and of its rate of convergence, comprehensive numerical solutions are presented that elucidate the action of the system and the effects of the various parameters involved. The parameters varied include the flexibility of the wall, the condition of top support, and the characteristics of the ground motion. The effects of both harmonic base motions and an actual earthquake record are examined. Special attention is paid to the effects of long-period, effectively static excitations. A maximum dynamic response is then expressed as the product of the corresponding static response and an appropriate amplification or deamplification factor. The response quantities examined include the displacements of the wall relative to the moving base, the dynamic wall pressures, and the total wall force, base shear and base moment.
\end{abstract}


 


\section{TABLE OF CONTENTS}

Section $\quad$ Page

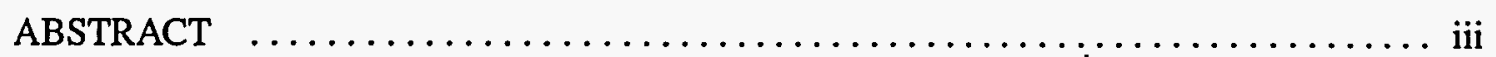

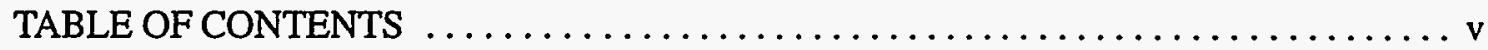

LIST OF TABLES $\ldots \ldots \ldots \ldots \ldots \ldots \ldots \ldots \ldots \ldots \ldots \ldots \ldots \ldots \ldots \ldots \ldots \ldots \ldots \ldots$

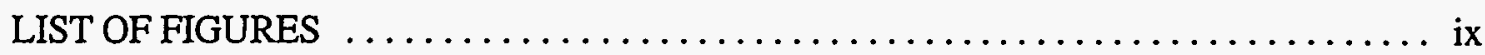

EXECUTIVE SUMMARY $\ldots \ldots \ldots \ldots \ldots \ldots \ldots \ldots \ldots \ldots \ldots \ldots \ldots \ldots \ldots \ldots \ldots \ldots$

ACKNOWLEDGMENT $\ldots \ldots \ldots \ldots \ldots \ldots \ldots \ldots \ldots \ldots \ldots \ldots \ldots \ldots \ldots \ldots \ldots \ldots \ldots \ldots$

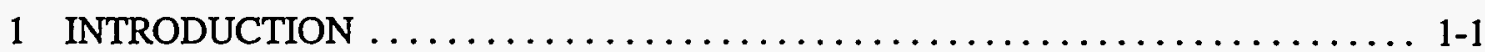

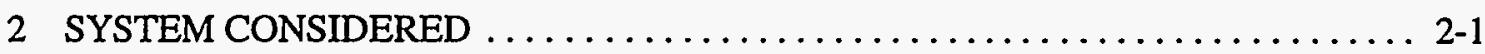

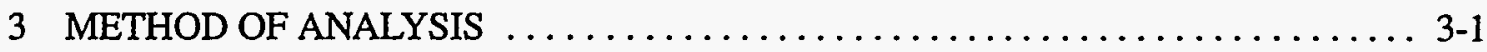

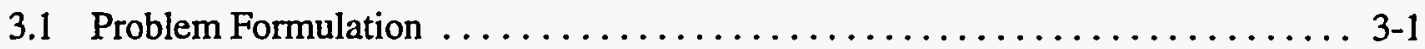

3.2 Evaluation of Generalized Forces $\ldots \ldots \ldots \ldots \ldots \ldots \ldots \ldots \ldots \ldots \ldots \ldots \ldots \ldots \ldots .2$

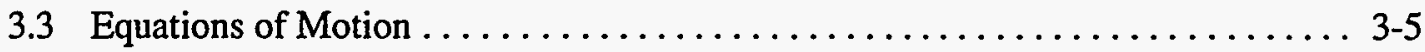

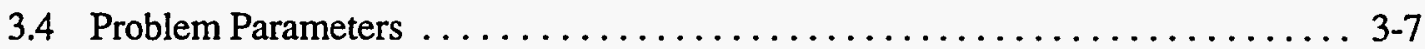

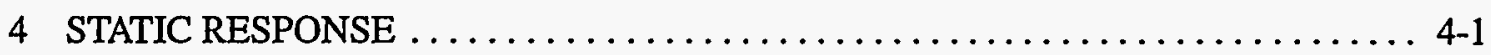

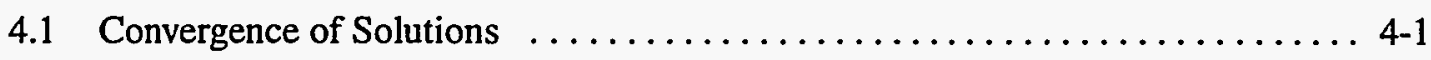

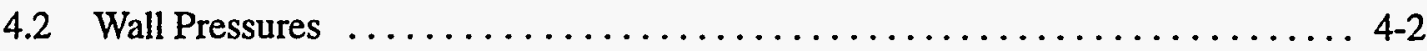

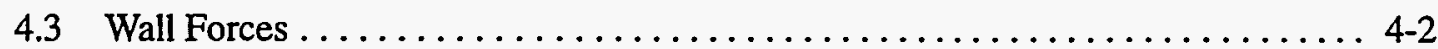

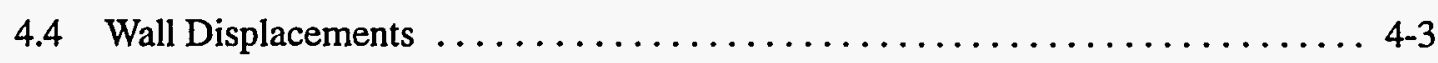

5 HARMONIC RESPONSE ........................ $5-1$

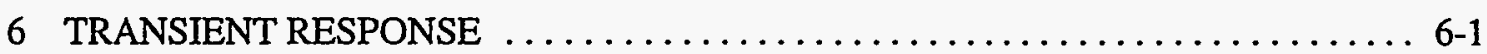

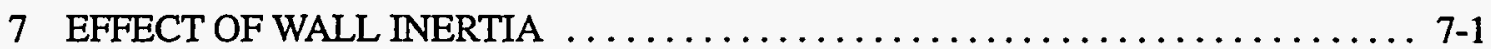

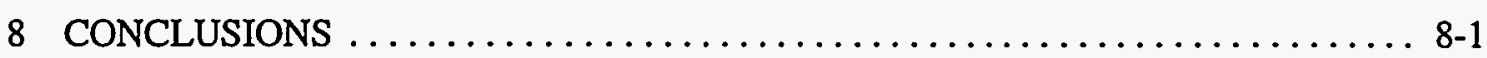

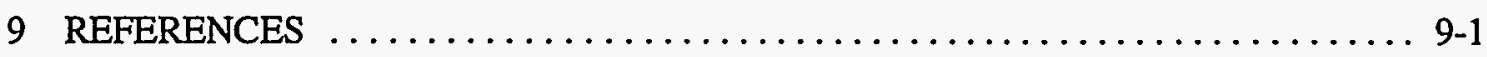

10 APPENDIX. NATURAL MODES AND INNER PRODUCTS $\ldots \ldots \ldots \ldots \ldots \ldots \ldots$

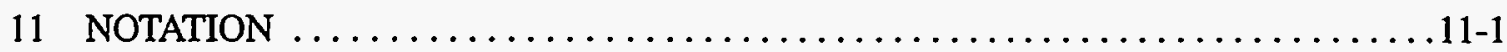





\section{LIST OF TABLES}

Table

Page

4.1 Normalized 'static' values of total wall force $\mathrm{P}_{\mathrm{st}}$, centroidal height $\mathrm{h}_{\mathrm{st}}$, base shear $\left(V_{b}\right)_{s t}$, base moment $\left(M_{b}\right)_{s t}$, and of displacement factors $c_{1}$ and $c_{2}$ for clamped-free $(\mathrm{C}-\mathrm{F})$ and clamped-hinged $(\mathrm{C}-\mathrm{H})$ walls $\ldots \ldots \ldots \ldots \ldots \ldots \ldots \ldots$

10.1 Dimensionless factors $\lambda_{j}$ and $\alpha_{j}$ in expressions for natural modes of vibration of clamped-free $(\mathrm{C}-\mathrm{F})$ and clamped-hinged $(\mathrm{C}-\mathrm{H})$ flexural beams $\ldots \ldots \ldots \ldots \ldots \ldots$ 10-3 


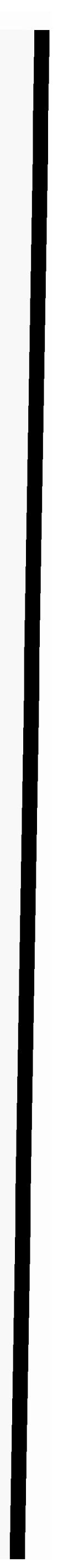




\section{LIST OF FIGURES}

\section{Figure}

Page

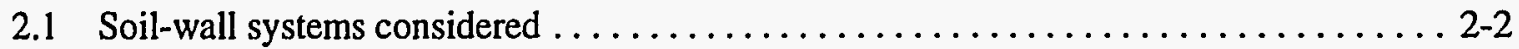

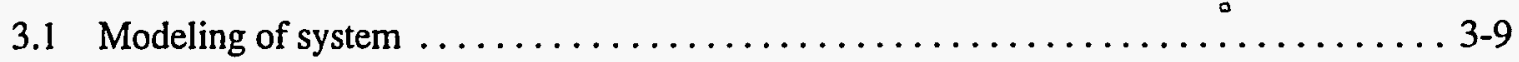

4.1 Convergence of wall displacements and pressures for statically excited systems with C-F

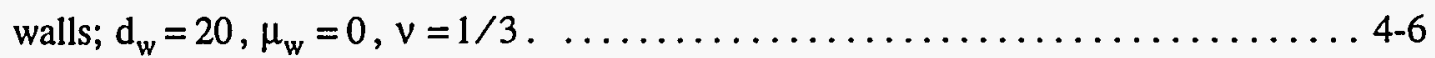

4.2 Convergence of base shear and base moment in wall of statically excited systems; C-F walls of different flexibilities, $\mu_{w}=0, v=1 / 3$

4.3 Convergence of base shear and base moment in wall of statically excited systems;

C-H walls of different flexibilities, $\mu_{w}=0, v=1 / 3$

4.4 Distributions of wall pressures for statically excited systems with different wall flexibilities;

$\mu_{w}=0, v=1 / 3$

4.5 Normalized values of total force per unit of wall length and of centroidal height for statically excited systems with different wall flexibilities; $\mu_{w}=0, v=1 / 3 \ldots \ldots$ 4-10

4.6 Normalized values of base shear and base moment per unit of wall length of statically excited systems; $C-H$ walls of different flexibilities, $\mu_{w}=0, v=1 / 3 \ldots \ldots$ 4-11

4.7 Distributions of wall displacements relative to base for statically excited systems with different wall flexibilities; $\mu_{w}=0, v=1 / 3 \ldots \ldots \ldots \ldots \ldots \ldots \ldots \ldots \ldots \ldots \ldots \ldots \ldots \ldots \ldots \ldots \ldots .12$

4.8 Coefficients $c_{1}$ and $c_{2}$ in expressions of maximum wall displacements relative to base for statically excited systems with different wall flexibilities; $\mu_{w}=0, v=1 / 3 \ldots$ 4-13

5.1 Frequency response curves for base shear per unit of wall length of harmonically excited systems; C-F walls of different flexibilities, $\mu_{w}=0, \delta_{w}=0.04$, $v=1 / 3, \delta=0.1$

5.2 Maximum amplification factor for total force in wall of harmonically excited systems with different wall flexibilities; $\mu_{w}=0, \delta_{w}=0.04, v=1 / 3, \delta=0.1 \ldots \ldots \ldots \ldots .4$ 
6..1 Normalized values of maximum total force per unit of wall length for systems with different wall flexibilities subjected to El Centro earthquake record; $\mu_{w}=0$, $\delta_{w}=0.04, v=1 / 3, \delta=0.1$

6.2 Amplification factors for total force in wall of systems with different wall flexibilities subjected to the El Centro earthquake record; $\mu_{w}=0, \delta_{w}=0.04, v=1 / 3, \delta=0.1 \ldots 6-4$

6.3 Average amplification factors for total force in wall of systems subjected to El Centro earthquake record; $\mu_{\mathrm{w}}=0, \delta_{\mathrm{w}}=0.04, v=1 / 3, \delta=0.1 ; A F$ averaged over period range $T_{1}=0.1$ to $0.5 \mathrm{sec}$

6.4 Normalized centroidal heights for systems with different wall flexibilities subjected to El Centro earthquake record; $\mu_{w}=0, \delta_{w}=0.04, v=1 / 3, \delta=0.1 \ldots \ldots \ldots \ldots 6-6$

7.1 Effective wall mass for statically excited systems with different wall flexibilities; $v=1 / 3$ 


\section{EXECUTIVE SUMMARY}

The study reported here is the sixth in a series of investigations of the response to ground shaking of retaining walls and deeply embedded vertical cylindrical structures. The objectives of these studies have been to provide insights into the dynamic responses of these systems and to formulate rational but simple methods for their analysis and design. The previous studies were described in Brookhaven National Laboratory reports 52357, 52372, 52402, 52444 and 52502.

Excepting limit-state analyses, in which the wall is considered to displace sufficiently at the base to mobilize the full shearing strength of the backfill, past analyses of the dynamic linear response of retaining walls dealt primarily with non-deflecting, rigid walls. In a recent contribution by the authors, it has been shown that, for walls that are rigid but elastically constrained against rotation at their base, both the magnitudes and distributions of the dynamic wall pressures and forces are quite sensitive to the flexibility of the base constraint and that, for realistic base flexibilities, these effects may be significantly lower than those computed for non-deflecting, rigid walls. Comparable effects also are expected for walls that are themselves flexible.

The purpose of this study is twofold: (1) to formulate a method of analysis with which the response to horizontal ground shaking of flexible walls retaining a uniform, linear viscoelastic stratum may be evaluated reliably and simply; and (2) to make a critical evaluation of the effects of wall flexibility on the magnitudes and distributions of the resulting wall pressures, forces and displacements.

The retained stratum in the reported solutions is considered to be of constant thickness and infinite extent in the horizontal direction, and the walls are considered to be fixed against both deflection and rotation at the base and to be either free or simply supported at the top. The support points of the wall and the base of the soil stratum are presumed to be excited by a space-invariant horizontal motion. In addition to the wall flexibility, the factors investigated include the properties of the stratum, and the characteristics of the base excitation. Both harmonic and earthquake-induced ground motions are examined. Special attention is paid to the effects of long-period, effectively static excitations. A maximum response for the dynamically excited system is then expressed as the product of the corresponding long-period, static response and an appropriate amplification or deamplification factor. After describing the method of analysis and discussing the rates of convergence of the resulting solutions, comprehensive numerical data are presented which elucidate the underlying response mechanisms and the effects and relative importance of the parameters involved. 
The principal conclusions of the study may be summarized as follows:

1. With the method of analysis and the numerical data presented, the dynamic response of the class of flexible retaining walls examined may be evaluated readily and with high degree of accuracy. The method, which makes use of Lagrange's equations of motion in combination with a recently proposed model for the action of soil-wall systems, is expected to prove of value in the analysis of a number of other problems as well.

2. The magnitudes and distributions of the wall displacements, wall pressures and associated forces induced by horizontal ground shaking in the systems examined are quite sensitive to the flexibility of the wall. Increasing this flexibility reduces the horizontal extensional stiffness of the retained medium relative to its shearing stiffness, and this reduction decreases the proportion of the soil inertia forces that gets transferred to the wall and, hence, the forces developed in it.

3. For realistic wall flexibilities, the total wall force or base shear for cantilever walls may well be less than one-half of that obtained for fixed-based, rigid walls, with the reduction in the base moment being even larger. Because of the greater effective stiffness of top-supported walls, the corresponding reductions for such walls are significantly smaller than for the cantilever systems.

4. Even for the $1940 \mathrm{El}$ Centro earthquake ground motion record, the maximum wall displacement relative to the moving base for cantilever walls of realistic flexibilities is found to be less than the values of 0.1 to 0.4 percent of the wall height normally accepted as the minimum required to develop a limit state in the backfill material.

5. The comprehensive numerical solutions presented and their analysis provide not only valuable insights into the effects and relative importance of the numerous factors that influence the response of the systems examined, but also a sound framework for assessing the behavior of even more complex soil-wall systems. 


\section{ACKNOWLEDGMENT}

This study was carried out at Rice University in cooperation with Brookhaven National Laboratory (BNL). The authors are grateful to the Department of Energy Program Directors and staff members John Tseng, James Antizzo, Dinesh Gupta, Kenneth Lang, David Pepson and Owen Thompson for supporting the study, and to Dr. Morris Reich of BNL for his understanding project management. Comments received from colleagues of BNL's Tank Seismic Experts Panel are also acknowledged with thanks. 


\section{SECTION 1}

\section{INTRODUCTION}

Despite the multitude of studies that have been carried out over the years, the dynamic response of retaining walls is far from being well understood. There is, in particular, a paucity of conclusive information that may be used in design applications.

Excepting limit-state analyses, ${ }^{1,2,3}$ in which the wall is considered to displace sufficiently at the base to mobilize the full shearing strength of the backfill, past analyses of the dynamic linear response of such systems dealt primarily with non-deflecting, rigid walls. ${ }^{4-7}$ Only exploratory studies have been made of flexible cantilever walls, ${ }^{8,9}$ and the limited numerical data presented by Sun and $\mathrm{Lin}^{9}$ are believed to be in error. In particular, in their expressions for the pressures induced on rigid walls, the factor $\varepsilon^{3}$ should appear without the exponent. More detailed accounts of previous analytical and experimental studies of retaining walls have been presented by Nazarian and Hadjian, ${ }^{10}$ Prakash, ${ }^{11}$ Whitman, ${ }^{12}$ and Veletsos and Younan. ${ }^{13}$

In a recent contribution by the authors, ${ }^{14}$ it has been shown that, for walls that are rigid but elastically constrained against rotation at their base, both the magnitudes and distributions of the dynamic wall pressures and forces are quite sensitive to the flexibility of the base constraint and that, for realistic base flexibilities, these effects may be significantly lower than those computed for non-deflecting, rigid walls. Comparable effects also are expected for walls that are themselves flexible.

The purpose of this study is twofold: (1) to formulate a method of analysis with which the response to horizontal ground shaking of flexible walls retaining a uniform, linear viscoelastic stratum may be evaluated reliably and simply; and (2) to make a critical evaluation of the effects of wall flexibility on the magnitudes and distributions of the resulting wall pressures, forces and displacements.

The retained stratum in the reported solutions is considered to be of constant thickness and infinite extent in the horizontal direction, and the walls are considered to be fixed against both defiection and rotation at the base and either free or simply supported at the top. The support points of the wall and the base of the soil stratum are presumed to be excited by a space-invariant horizontal motion. In addition to the wall flexibility, the factors investigated include the properties of the stratum and the characteristics of the base excitation. Both harmonic and earthquake-induced ground motions are 
examined. Special attention is paid to the effects of long-period, effectively static excitations. A maximum response for the dynamically excited system is then expressed as the product of the corresponding long-period, static response and an appropriate amplification or deamplification factor. After describing the method of analysis and discussing the rates of convergence of the resulting solutions, comprehensive numerical data are presented which elucidate the underlying response mechanisms and the effects and relative importance of the parameters involved. 


\section{SECTION 2}

\section{SYSTEM CONSIDERED}

The systems examined are shown in Figure 2.1. They consist of a semiinfinite, uniform layer of viscoelastic material that is free at its upper surface, is bonded to a non-deformable, rigid base, and is retained along one of its vertical boundaries by a uniform, flexible wall. The wall is considered to be fixed against both deflection and rotation at the base and to be either free or hinged at the top. The clamped-free and clamped-hinged systems are identified with the symbols $\mathrm{C}-\mathrm{F}$ and $\mathrm{C}-\mathrm{H}$, respectively. The free boundary is representative of cantilever retaining walls, whereas the hinged or simply supported boundary is more nearly representative of the support condition for basement walls. The support points of the wall and the base of the soil stratum are presumed to experience a spaceinvariant horizontal motion, the acceleration of which at any time $t$ is $\ddot{x}_{g}(t)=\ddot{x}_{g}$. Material damping for both the medium and the wall is considered to be of the constant hysteretic type.

The properties of the soil stratum are defined by its mass density $\rho$, shear modulus of elasticity $G$, Poisson's ratio $v$, and material damping factor $\delta$, which is considered to be the same for both shearing and axial deformations. The factor $\delta$ is the same as the $\tan \delta$ factor used by the second author and his associates in studies of foundation dynamics and soil-structure interaction ${ }^{15,16}$ and twice as large as the percentage of critical damping $\beta$ used by other authors in related studies. ${ }^{17,18}$ The properties of the wall are defined by its thickness $t_{w}$, mass per unit of surface area $\mu_{w}$, Young's modulus of elasticity $E_{w}$, Poisson's ratio $v_{w}$, and damping factor $\delta_{w}$ which, like $\delta$, is twice as large as the corresponding percentage of critical damping.

The wall displacements relative to the moving base and the resulting wall pressures and forces for a base-excited system can be shown to be identical to those induced in a force-excited system for which the base is stationary and the stratum and wall are subjected to lateral body forces of intensity $-\rho \ddot{x}_{g}(t)$ and $-\mu_{w} \ddot{x}_{g}(t)$, respectively. For excitations with dominant frequencies that are very low compared to the fundamental frequency of the stratum, the action of a force-excited system may be easier to visualize than that of the base-excited system. 


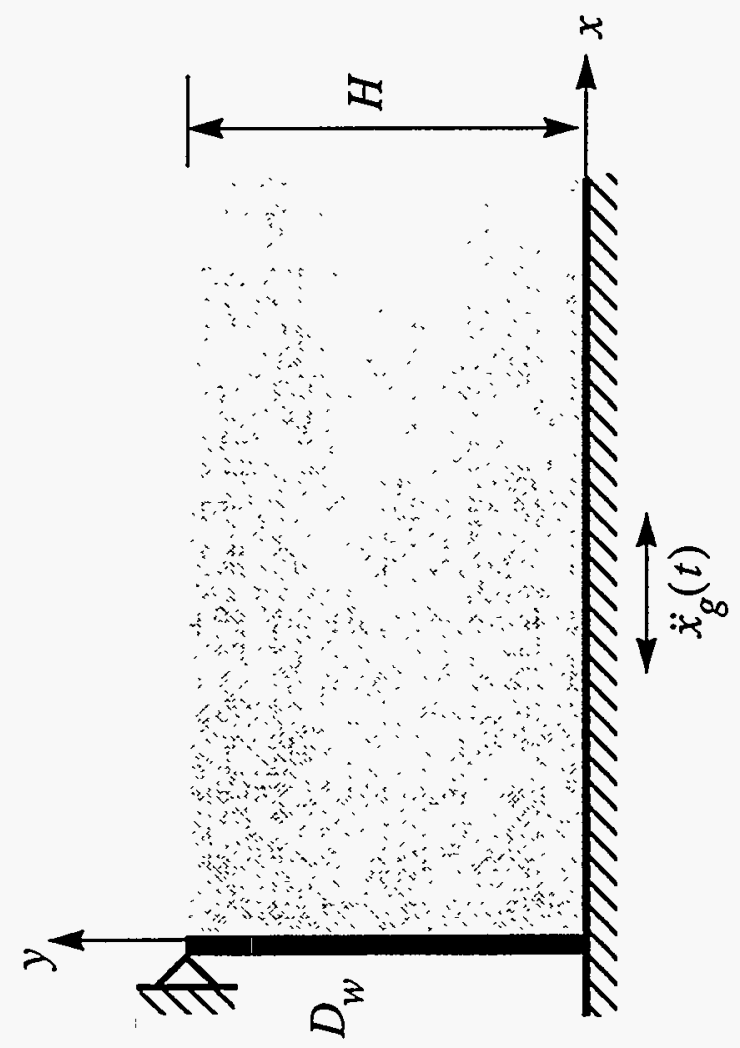

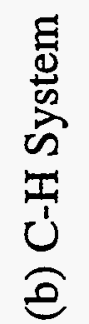

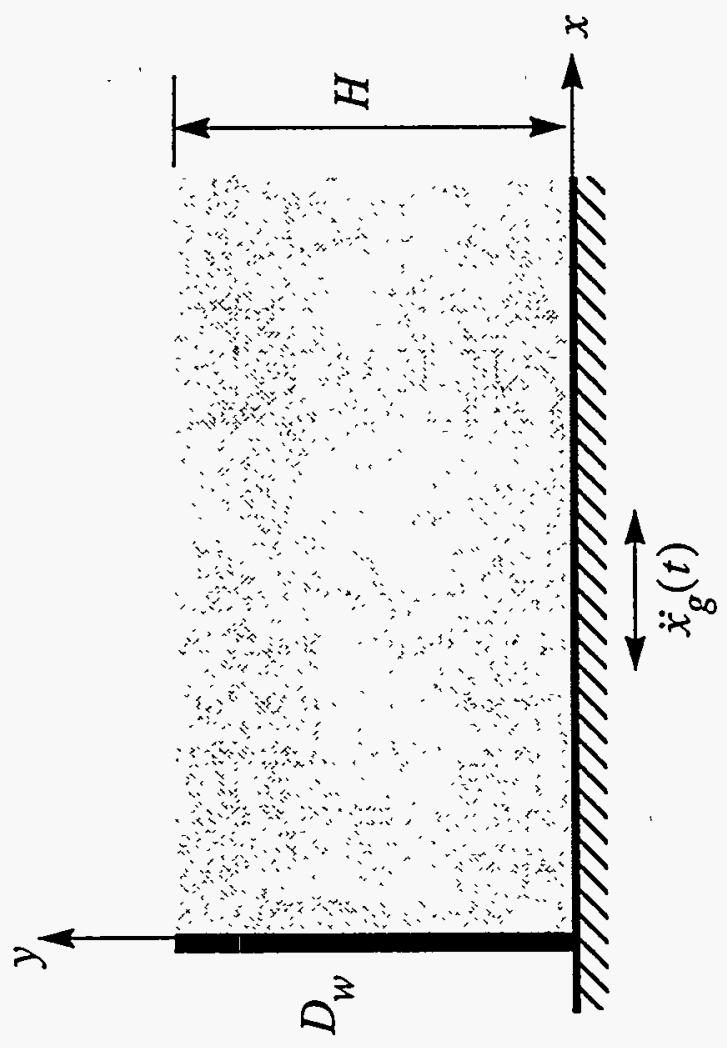

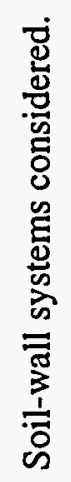

굴 


\section{SECTION 3}

\section{METHOD OF ANALYSIS}

The method of analysis employed is an extension of that used in previous studies by the authors. ${ }^{14}$ Fundamental to this method is the assumption that, under the horizontal excitation considered, no vertical normal stresses develop anywhere in the medium, i.e. $\sigma_{y}=0$. It is further assumed that there is complete bonding between the wall and the retained medium, and that the horizontal variations of the vertical displacements of the medium are negligible so that the horizontal shearing stresses $\tau_{x y}$ can be expressed as $\tau_{x y}=G^{*}(\partial u / \partial y)$, where $u$ is the horizontal displacement of an arbitrary point of the medium relative to the moving base, $G^{*}=G(1+i \delta)$ is the complex-valued shear modulus, and $i=\sqrt{-1}$.

\subsection{Problem Formulation}

The instantaneous value of the wall displacement relative to the moving base, $w(\eta, t)$, is expressed as a linear combination of the natural modes of vibration of an appropriately supported, uniform, flexural beam as

$$
w(\eta, t)=\sum_{j=1}^{J} \phi_{j}(\eta) q_{j}(t)
$$

where $\eta=y / H$ is a dimensionless measure of the vertical distance $y$ from the base; $\phi_{j}(\eta)$ is the $j$ th natural mode of vibration of the beam; $q_{j}(t)$ is a generalized coordinate defining the degree of participation of the $j$ th mode, $\phi_{\mathbf{j}}(\eta)$, at any time $t$; and $J$ is the total number of modes considered. For a wall that is free at the top, $\phi_{j}(\eta)$ refers to the $j$ th mode of a C-F beam, whereas for a wall that is simply supported at the top, it refers to the corresponding mode of a $\mathrm{C}-\mathrm{H}$ beam. The expressions for these modes are given in the Appendix.

The equations of motion for the system are obtained by repeated application of Lagrange's equation ${ }^{19}$

$$
\frac{d}{d t}\left\{\frac{\partial T_{w}}{\partial \dot{q}_{j}}\right\}-\frac{\partial T_{w}}{\partial q_{j}}+\frac{\partial V_{w}}{\partial q_{j}}=F_{j} \quad j=1,2, \ldots, J
$$

where $T_{w}$ is the kinetic energy of the wall; $V_{w}$ is its strain energy; $F_{j}$ is the $j$ th generalized force induced by the soil pressures on the wall; and a dot superscript denotes a differentiation with respect to time. The kinetic and strain energies are given by 


$$
\begin{gathered}
\mathrm{T}_{\mathrm{w}}=\frac{1}{2} \mu_{\mathrm{w}} \mathrm{H} \int_{0}^{1}\left(\dot{\mathrm{x}}_{\mathrm{g}}+\dot{\mathrm{w}}\right)^{2} \mathrm{~d} \eta \\
\mathrm{V}_{\mathrm{w}}=\frac{1}{2} \frac{\mathrm{D}_{\mathrm{w}}^{*}}{\mathrm{H}^{3}} \int_{0}^{1}\left(\mathrm{w}^{\prime \prime}\right)^{2} \mathrm{~d} \eta
\end{gathered}
$$

in which $\dot{x}_{\mathrm{g}}$ is the instantaneous value of the ground velocity; $\dot{w}$ is the corresponding wall velocity relative to the moving base; a prime superscript denotes a differentiation with respect to $\eta$; and $D_{w}^{*}=D_{w}\left(1+i \delta_{w}\right)$ is the complex-valued flexural rigidity of the wall, with $D_{w}$ given by

$$
D_{w}=\frac{E_{w} t_{w}^{3}}{12\left(1-v_{w}^{2}\right)}
$$

On substituting equations (3) and (4) into equation (2) and making use of equation (1), Lagrange's equations reduce to

$$
\mu_{w} H\left\langle\phi_{j}, \phi_{j}\right\rangle \ddot{q}_{j}+\frac{D_{w}^{*}}{H^{3}}\left\langle\phi_{j}^{\prime \prime}, \phi_{j}^{\prime \prime}\right\rangle q_{j}=F_{j}-\mu_{w} H\left\langle\phi_{j}, 1\right\rangle \ddot{x}_{g} \quad j=1,2, \ldots, J
$$

in which $\langle a, b\rangle$ denotes the integral over the interval $[0,1]$ of the product of the two bracketed functions.

\subsection{Evaluation of Generalized Forces}

The as yet undetermined generalized forces $F_{j}$ are the coefficients in an expansion of the variation of the work done by the soil pressures, $\delta \tilde{W}$, by variations in the generalized coordinates $\delta q_{j}$, i.e.,

$$
\delta \tilde{W}=\sum_{j=1}^{J} F_{j} \delta q_{j}
$$

These forces are determined as follows. First, the normal pressures exerted by the soil on the wall, $\sigma(\eta, t)$, are expressed as the sum of two components as

$$
\sigma(\eta, t)=\sigma_{r}(\eta, t)+\sigma_{f}(\eta, t)
$$

where $\sigma_{\mathrm{r}}$ represents the component associated with a non-deflecting rigid wall, and $\sigma_{\mathrm{f}}$ represents the component associated with the wall flexibility. The work $\tilde{W}$ may then be expressed as

$$
\tilde{W}=H\left\langle\sigma_{r}, w\right\rangle+\frac{H}{2}\left\langle\sigma_{f}, w\right\rangle
$$

Inasmuch as the pressure component $\sigma_{\mathrm{r}}$ is considered to pre-exist and is independent of the wall displacement, the factor $1 / 2$ is not needed in front of the first term. The sign convention for pressures and displacements is that used in theory of elasticity. Specifically, displacements are considered to be positive when inward, i.e., when directed along the positive $\mathrm{x}$-axis, and normal stresses are positive when tensile. On taking the variation of $\tilde{W}$, recalling that $\sigma_{\mathrm{r}}$ is independent of $w$, and making use of 
equation (8), the $j$ th generalized force $F_{j}$ is expressed as

$$
F_{j}=H\left\langle\sigma_{r}, \frac{\partial w}{\partial q_{j}}\right\rangle+\frac{H}{2}\left\{\left\langle\sigma_{f}, \frac{\partial w}{\partial q_{j}}\right\rangle+\left\langle\frac{\partial \sigma_{f}}{\partial q_{j}}, w\right\rangle\right\}
$$

The stress components in the above expressions are evaluated by application of the model for the soilwall system proposed earlier by the authors. ${ }^{14}$ Shown in Figure 3.1, the wall in this model is considered to be connected by a set of springs and dashpots to a cantilever shear-beam having the properties of the retained medium. The shear-beam represents the action of the soil stratum at the farfield while the springs and dashpots, which have frequency-dependent properties, simulate the restraining action of the medium between the far field and the wall. Strictly speaking, the model is used to evaluate the response of the system to a harmonic excitation. The response to an arbitrary transient excitation is then determined by application of Fourier transform techniques.

For a harmonic base motion with an acceleration

$$
\ddot{x}_{g}(t)=\ddot{X}_{g} e^{i \omega t}
$$

in which $\ddot{X}_{g}$ is the acceleration amplitude and $\omega$ is the circular frequency of the motion, the horizontal displacement of the shear-beam relative to the moving base at an arbitrary elevation and time, $u_{s}(\eta, t)$, may be expressed as

$$
u_{s}(\eta, t)=\sum_{n=1}^{N} U_{n} \Psi_{n}(\eta) e^{i \omega t}
$$

where $\Psi_{n}$ is the $n$th natural mode of vibration of the shear-beam, given by

$$
\Psi_{n}(\eta)=\sin \left[\frac{(2 n-1) \pi}{2} \eta\right]
$$

$\mathrm{U}_{\mathrm{n}}$ and $\omega_{\mathrm{n}}$ are the corresponding participation factor and circular natural frequency, given by

$$
U_{\mathrm{n}}=-\frac{16}{\pi^{3}} \frac{\rho \ddot{X}_{\mathrm{g}} \mathrm{H}^{2}}{\mathrm{G}} \frac{1}{(2 \mathrm{n}-1)^{3}} \frac{1}{1-\left(\omega / \omega_{\mathrm{n}}\right)^{2}+\mathrm{i} \delta}
$$

and

$$
\omega_{\mathrm{n}}=\frac{(2 \mathrm{n}-1) \pi}{2} \frac{\mathrm{v}_{s}}{\mathrm{H}}
$$

respectively, $v_{s}=\sqrt{G / p}$ is the shear-wave velocity for the medium, and $N$ is a sufficiently large integer representing the total number of modes considered. If the displacement of the wall is expressed similarly in terms of the natural modes of the shear-beam as 


$$
w(\eta, t)=\sum_{n=1}^{N} W_{n} \Psi_{n}(\eta) e^{i \omega t}
$$

where $\mathrm{W}_{\mathrm{n}}$ are participation factors that remain to be determined, then the total dynamic wall pressure $\sigma(\eta, t)$ may be computed from ${ }^{14}$

$$
\sigma(\eta, t)=\sum_{n=1}^{N} K_{n}\left(U_{n}-W_{n}\right) \Psi_{n}(\eta) e^{i \omega t}
$$

The quantity $K_{n}$ in this expression represents the complex-valued impedance or dynamic stiffness of the spring-dashpot combination when both the shear-beam and the wall vibrate in the $n$th shear-beam mode $\Psi_{n}(\eta)$, and it is given by ${ }^{14}$

$$
\mathrm{K}_{\mathrm{n}}=\frac{(2 \mathrm{n}-1) \pi}{2} \sqrt{\frac{2}{1-v}} \frac{\mathrm{G}}{\mathrm{H}} \cdot \sqrt{(1+\mathrm{i} \delta)\left[1-\left(\omega / \omega_{\mathrm{n}}\right)^{2}+\mathrm{i} \delta\right]}
$$

Incidentally, the sum of the terms in equation (17) involving the factors $U_{n}$ represents the pressure component $\sigma_{\mathrm{r}}$ for a rigid wall, whereas the sum of the terms involving the factors $W_{n}$ represents the pressure component $\sigma_{\mathrm{f}}$ associated with the wall flexibility.

It is important to note that the expansion defined by equation (16) is used only for the evaluation of the wall pressures, the wall displacements being determined from equation (1). This expansion is possible because the shear-beam modes constitute a complete set of functions in the interval $[0,1]$ and may, therefore, be used as a basis for the representation of any function in that interval. ${ }^{20}$

The participation factors $\mathrm{W}_{\mathrm{n}}$ in equation (16) are determined by expanding the $j$ th mode of vibration of the wall $\phi_{j}(\eta)$ in terms of the shear-beam modes $\psi_{n}(\eta)$. On making use of the orthogonality property of the latter, one obtains

$$
\phi_{j}(\eta)=\sum_{n=1}^{N} \frac{\left\langle\phi_{j}, \psi_{n}\right\rangle}{\left\langle\psi_{n}, \psi_{n}\right\rangle} \psi_{n}(\eta)
$$

and on noting that for the harmonic response considered, the generalized coordinates $q_{j}$ are of the form

$$
q_{j}(t)=Q_{j} e^{i \omega t}
$$

equation (1) may be written as

$$
w(\eta, t)=\sum_{j=1}^{J}\left\{\sum_{n=1}^{N} \frac{\left\langle\phi_{j}, \psi_{n}\right\rangle}{\left\langle\psi_{n}, \Psi_{n}\right\rangle} \Psi_{n}(\eta)\right\} Q_{j} e^{i \omega t}
$$

or as 


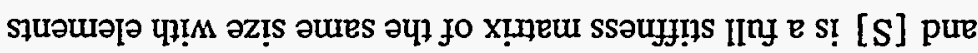

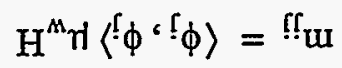

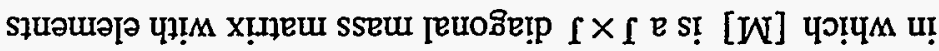

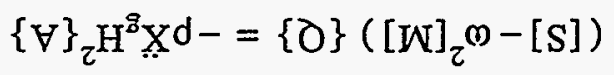

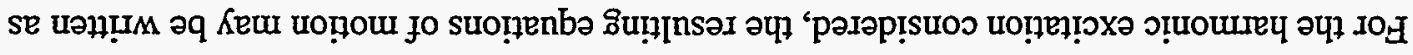

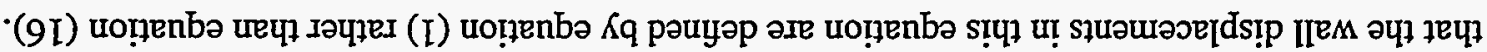

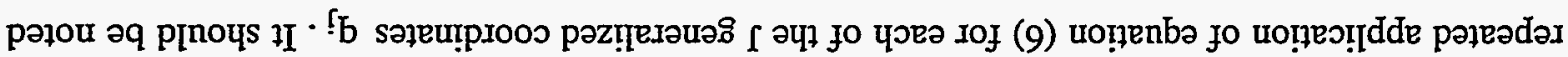

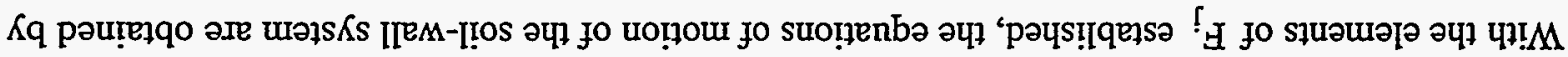

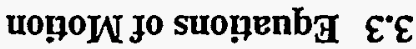

(9乙)

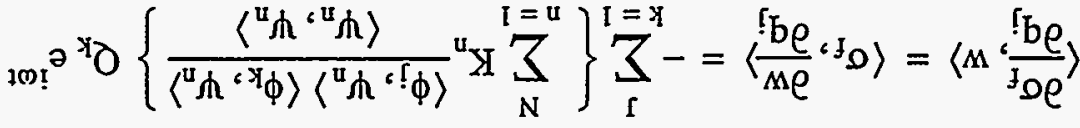

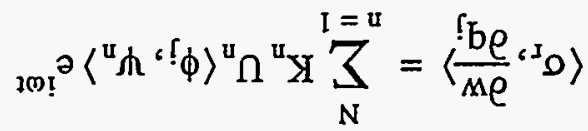

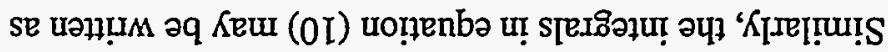

$(† \tau)$

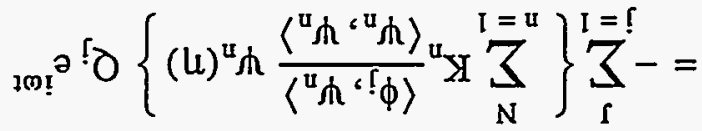

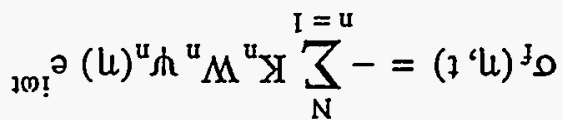

pure

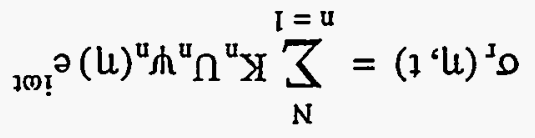

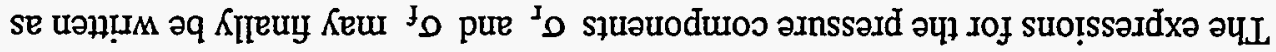

(乙乙)

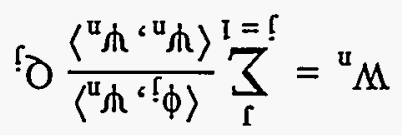

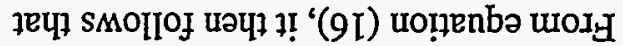

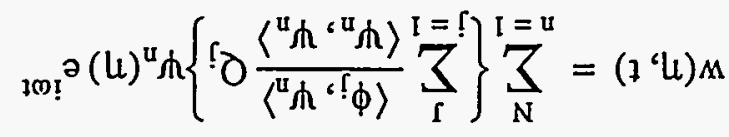




$$
s_{j k}=\left\langle\phi_{j}^{\prime \prime}, \phi_{k}^{\prime \prime}\right\rangle \frac{D_{w}^{*}}{H^{3}} \delta_{j k}+H \sum_{n=1}^{N} \frac{\left\langle\phi_{j}, \psi_{n}\right\rangle\left\langle\phi_{k}, \psi_{n}\right\rangle}{\left\langle\psi_{n}, \psi_{n}\right\rangle} K_{n}
$$

where $\delta_{\mathrm{jk}}$ is the Kronecker delta

$$
\delta_{\mathrm{jk}}= \begin{cases}0 & \mathrm{j} \neq \mathrm{k} \\ 1 & \mathrm{j}=\mathrm{k}\end{cases}
$$

Finally, $\{Q\}$ is the vector of the amplitudes of the generalized coordinates $Q_{j}$, and $\{A\}$ is a vector of normalized exciting forces with elements

$$
a_{j}=\left\langle\phi_{j}, 1\right\rangle \frac{\mu_{w}}{\rho H}-\frac{1}{\rho \ddot{X}_{g} H} \sum_{n=1}^{N}\left\langle\phi_{j}, \Psi_{n}\right\rangle K_{n} U_{n}
$$

With the vector $\{Q\}$ determined from the solution of the system of algebraic equations (28), the generalized coordinates $q_{j}$ are determined from equation (20), the wall displacement are determined from equation (1), and the wall pressures are determined from equation (8) by making use of equations (24) and (25). The total dynamic wall force, $P(t)$, and the moment of this force about the base, $\tilde{\mathrm{M}}(\mathrm{t})$, are finally determined by appropriate integrations of the wall pressures and associated inertia forces. The results are

$$
\begin{aligned}
& \mathrm{P}(\mathrm{t})=\mathrm{H}\langle\sigma, 1\rangle-\mu_{\mathrm{w}} \mathrm{H} \ddot{x}_{\mathrm{g}}(\mathrm{t})-\mu_{\mathrm{w}} \mathrm{H}\langle\ddot{\mathrm{w}}, 1\rangle \\
& =H \sum_{n=1}^{N} K_{n}\left\{U_{n}-\sum_{j=1}^{J} \frac{\left\langle\phi_{j}, \Psi_{n}\right\rangle}{\left\langle\psi_{n}, \Psi_{n}\right\rangle} Q_{j}\right\}\left\langle\Psi_{n}, 1\right\rangle e^{i \omega t} \\
& -\mu_{w} \ddot{X}_{g} H e^{i \omega t}+\omega^{2} \mu_{w} H \sum_{j=1}^{J}\left\langle\phi_{j}, 1\right\rangle Q_{j} e^{i \omega t} \\
& \tilde{M}(t)=H^{2}\langle\sigma, \eta\rangle-\frac{1}{2} \mu_{w} H^{2} \ddot{x}_{g}(t)-\mu_{w} H^{2}\langle\ddot{w}, \eta\rangle \\
& =H^{2} \sum_{n=1}^{N} K_{n}\left\{U_{n}-\sum_{j=1}^{J} \frac{\left\langle\phi_{j}, \psi_{n}\right\rangle}{\left\langle\psi_{n}, \psi_{n}\right\rangle} Q_{j}\right\}\left\langle\Psi_{n}, \eta\right\rangle e^{i \omega t} \\
& -\frac{1}{2} \mu_{w} \ddot{X}_{g} H^{2} e^{i \omega t}+\omega^{2} \mu_{w} H^{2} \sum_{j=1}^{J}\left\langle\phi_{j}, \eta\right\rangle Q_{j} e^{i \omega t}
\end{aligned}
$$

For a clamped-free wall, the force $P(t)$ is clearly equal to the base shear in the wall, $V_{b}(t)$, and the overturning base moment $\tilde{M}(t)$ also equals the corresponding wall moment, $M_{b}(t)$. For the clampedhinged wall, the base wall moment is determined by differentiation of the wall displacement, i.e., from

$$
\mathrm{M}_{\mathrm{b}}(\mathrm{t})=\left.\frac{\mathrm{D}_{\mathrm{w}}^{*}}{\mathrm{H}^{2}} \mathrm{w}^{\prime \prime}\right|_{\eta=0}
$$

and the corresponding base shear is computed by considering the equilibrium of moments about the 
hinged support, i.e. from

$$
V_{b}(t)=P(t)-\frac{1}{H}\left[\tilde{M}(t)-M_{b}(t)\right]
$$

The latter expression converges faster than that obtained by triple differentiation of the wall displacement. Shears and moments are considered to be positive when induced by positive normal pressures.

The beam modes $\phi_{j}(\eta)$ for the two sets of boundary conditions examined and the expressions for the various integrals of these modes in the equations presented are given in the Appendix.

\subsection{Problem Parameters}

The primary parameters affecting the response of the system are the relative flexibility of the wall and retained medium, defined by

$$
\mathrm{d}_{\mathrm{w}}=\frac{G H^{3}}{\mathrm{D}_{\mathrm{w}}}
$$

the support condition of the wall at the top, and the characteristics of the base motion. For a harmonic excitation, the response is controlled by the frequency ratio $\omega / \omega_{1}$, where $\omega$ is the circular frequency of the base motion and of the resulting steady-state response, and $\omega_{1}$ is the fundamental circular natural frequency of the stratum when it is considered to act as an unconstrained, vertical cantilever shear-beam. For an arbitrary transient excitation, the relevant stratum property is its fundamental cyclic frequency $f_{1}=\omega_{1} / 2 \pi$, or its corresponding period $T_{1}=1 / f_{1}=2 \pi / \omega_{1}$, given by

$$
\mathrm{T}_{1}=\frac{4 \mathrm{H}}{\mathrm{v}_{\mathrm{s}}}
$$

Additional parameters are Poisson's ratio and the damping factor for the soil, $v$ and $\delta$, the damping factor for the wall $\delta_{w}$, and the ratio of mass densities for the wall and the retained medium $\mu_{w} / p H$.

As a measure of the range of $d_{w}$ values that may be encountered in practice, consider a concrete wall with $\mathrm{E}_{\mathrm{w}}=3 \times 10^{6} \mathrm{psi}, \mathrm{v}_{\mathrm{w}}=0.17$ and a height-to-thickness ratio $\mathrm{H} / \mathrm{t}_{\mathrm{w}}=10$ retaining a soil for which the unit weight $\gamma=\rho g=100 \mathrm{lb} / \mathrm{ft}^{3}$. On noting that

$$
\mathrm{d}_{\mathrm{w}}=\frac{G H^{3}}{D_{\mathrm{w}}}=12\left(1-v_{\mathrm{w}}^{2}\right) \frac{G}{\mathrm{E}_{\mathrm{w}}}\left(\frac{\mathrm{H}}{\mathrm{t}_{\mathrm{w}}}\right)^{3}
$$

and that $G=\rho v_{s}^{2}$, one finds that $d_{w}$ varies from 3.35 for a soil with $v_{s}=200 \mathrm{ft} / \mathrm{sec}$ to 30.2 for a soil with $v_{s}=600 \mathrm{ft} / \mathrm{sec}$.

For the solutions presented in the following sections, the wall, unless otherwise indicated, is considered to be massless (i.e., $\mu_{w}=0$ ); Poisson's ratio for the soil is taken as $v=1 / 3$; and the 
damping factors for the soil and wall are taken as $\delta=0.1$ and $\dot{\delta}_{w}=0.04$, respectively (i.e., as $5 \%$ and $2 \%$ of critical damping). The frequency ratio $\omega / \omega_{1}$ for harmonic motions is varied over a wide range, and so is the natural period $\mathrm{T}_{1}$ of the stratum for the earthquake ground motion. 


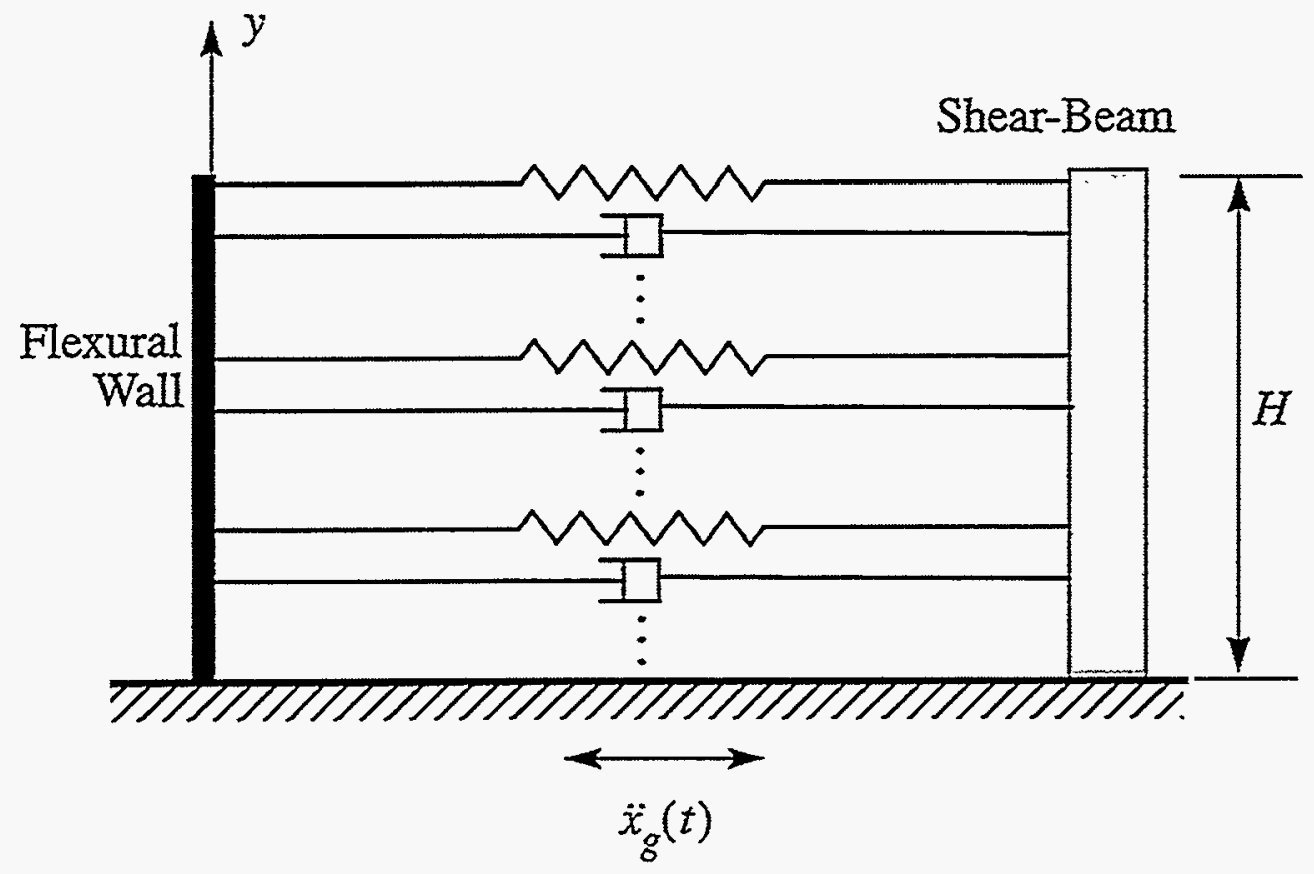

Figure 3.1 Modeling of system. 


\section{SECTION 4}

\section{STATIC RESPONSE}

It is desirable to begin by examining the responses obtained for excitations the dominant frequencies of which are extremely small compared to the fundamental natural frequency of the soil-wall system (i.e., for values of $\omega / \omega_{1} \rightarrow 0$ or $f_{1} \rightarrow \infty$ ). Such excitations and the resulting effects are referred to as 'static' and are identified with the subscript st. This term should not be confused with that normally used to represent the effects of gravity forces. In the equivalent, force-excited version of the problem referred to previously, the static excitation is represented by horizontal body forces of intensity $-\rho \ddot{X}_{g}$ for the retained medium and $-\mu_{\mathrm{w}} \ddot{X}_{\mathrm{g}}$ for the wall. A maximum dynamic effect for an arbitrary transient excitation is then expressed as the product of the corresponding static effect and an appropriate amplification or deamplification factor.

\subsection{Convergence of Solutions}

The accuracy of the solutions for the method of analysis presented clearly depends both on the number of flexural beam modes $J$ and on the number of shear-beam modes $N$ considered. Part (a) of Figure 4.1 shows the heightwise variations of the wall displacements computed for a cantilever (C-F) wall with $d_{w}=20$ for several different combinations of $J$ and $N$; and part (b) shows the variations of the corresponding wall pressures. Displacements are normalized with respect to $\rho \ddot{X}_{\mathrm{g}} \mathrm{H}^{2} / \mathrm{G}$ and pressures with respect to $\rho \ddot{X}_{\mathrm{g}} \mathrm{H}$. The wall in these solutions is presumed to be massless and Poisson's ratio for the retained medium is taken as $v=1 / 3$. Unless specifically otherwise indicated, the same conditions are presumed for all solutions that follow.

It is clear from Figure 4.1 that the number of shear-beam modes required for convergence is considerably larger than that of the flexural beam modes. Additionally, this number is much larger for wall pressures than for displacements. Considering that the exact distribution of wall pressures, unlike that of displacements, is so much different from that obtained with $\mathrm{J}=\mathrm{N}=1$, this result should not be surprising. The precise definition of the actual, smoothly varying wall pressures for the conditions examined here requires no more than 3 beam modes and no less than 50 shear-beam modes. The solutions in the following sections were obtained with this, or a somewhat larger, number of modes.

The upper part of Figure 4.2 shows the rates of convergence of the total wall force per unit of wall length, $P_{s t}$, or base shear per unit of wall length, $\left(V_{b}\right)_{s t}$, for cantilever (C-F) walls having several 
different values of the relative flexibility factor $d_{w}$. A normalized measure of this force is plotted as a function of the number of flexural beam modes $J$ and selected numbers of shear-beam modes. The corresponding information for the overturning base moment $\left(M_{b}\right)_{s t}$ is displayed in the lower part of the figure.

It is observed that the rates of convergence for these forces, particularly for the base moment, are much more rapid than for the wall pressures. Additionally, these rates decrease with increasing wall flexibility, and even for the largest values of $d_{w}$ considered, excellent results for both base shear and base moment are obtained with as few as 2 or 3 beam modes and 5 to 10 shear-beam modes.

Although strictly applicable to cantilever walls, the indicated trends also hold for walls that are simply supported at the top. This is demonstrated in Figure 4.3, which shows the corresponding plots for the base shear and bàse moment of top-constrained systems.

The convergence rates in the foregoing discussion were for a long-period, effectively static excitation. For dynamic excitations of the type associated with earthquake-induced ground motions, the convergence rates would be expected to be even better as the responses are likely to be influenced less by the contributions of the higher modes of vibration than are the corresponding static responses.

\subsection{Wall Pressures}

Figure 4.4 shows the precise distributions of the wall pressures for systems with values of $d_{w}$ in the range between zero and 40 . The plots on the left are for cantilever walls, whereas those on the right are for walls that are simply supported at the top. The other parameters for the systems examined are the same as those identified earlier.

It is observed that both the magnitudes and distributions of the pressures are quite sensitive to the flexibility of the wall and substantially different for the two sets of support considered. Increasing the wall flexibility reduces the horizontal extensional stiffness of the medium relative to its shearing stiffness, and this reduction, in turn, increases the proportion of the inertia forces transmitted by horizontal shearing action to the base, and decreases the proportion transmitted to the wall. For rigid walls $\left(d_{w}=0\right.$ ), the pressures increase almost as a quarter-sine from zero at the base to a maximum at the top, whereas for the flexible walls, there is a sharp change in the intensity of the pressure near the top, with the pressure increasing for the top-constrained system and decreasing and changing signs for the cantilever system.

\subsection{Wall Forces}

In the upper part of Figure 4.5, the static values of the total wall force, $P_{\text {st }}$, for both cantilever and topsupported walls are plotted as a function of the wall flexibility factor $d_{w}$. As might have been anticipated from the information on wall pressures presented earlier, an increase in wall flexibility 
reduces the wall force, the reduction being significantly larger for cantilever walls than for topconstrained walls. Considering that the effective flexibility of a cantilever wall of a specified value of $d_{w}$ is substantially larger than that of a top-constrained wall of the same $d_{w}$ value (for a pressure that increases as a quarter-sine from base to top, the ratio of the two flexibilities is 27), the latter trend should not be surprising.

In the lower part of Figure 4.5, the centroidal height, $h_{\text {st }}$, defined as the distance from the base to the point of application of the resultant wall force, is plotted normalized with respect to the height of the medium $H$ for each of the two support conditions considered. Note that, whereas for cantilever walls, the $h_{s t} / H$ ratio decreases from 0.6 for a rigid wall to less than 0.3 for walls of high flexibility, for the top-constrained walls, it has practically the constant value of 0.6 .

For the cantilever walls, $P_{s t}$ also represents the 'static' value of the base shear in the wall $\left(V_{b}\right)_{s t}$, and $h_{\text {st }}$ also represents the height by which $\mathrm{P}_{\text {st }}$ must be multiplied to yield the 'static' value of the base wall moment $\left(\mathrm{M}_{\mathrm{b}}\right)_{\mathrm{st}}$. These relationships do not, of course, hold true for top-constrained walls. In Figure 4.6, the base shear in the wall of such systems is plotted normalized with respect to the total wall force $P_{s t}$, and the corresponding base moment is plotted normalized with respect to $P_{s t} H$.

Normalized values of $P_{s t},\left(V_{b}\right)_{s t}$ and $\left(M_{b}\right)_{s t}$ for both cantilever and top-supported walls are also listed in Table 4.1, along with the corresponding centroidal heights $h_{s t}$.

\subsection{Wall Displacements}

In Figure 4.7, the displacement configurations for cantilever and top-constrained walls are plotted normalized to a unit peak value for values of the relative wall flexibility factor $d_{w}$ between zero and 40. It is observed that, within this range of $d_{w}$ values, the displacement configurations, particularly those for the top-supported walls, are insensitive to the value of $d_{w}$ involved. As $d_{w} \rightarrow 0$, the configurations naturally approach those obtained for a beam subjected to the pressures induced on a non-deflecting, rigid wall.

The maximum values of the wall displacements may be expressed either in terms of the wall properties as

$$
\left(w_{s t}\right)_{\max }=c_{1} \frac{P_{s t} H^{3}}{D_{w}}
$$

or, more conveniently, in terms of the properties of the retained medium as

$$
\left(w_{s t}\right)_{\max }=c_{2} \frac{\rho \ddot{X}_{g} H^{2}}{G}=c_{2} \frac{\ddot{X}_{g} H^{2}}{v_{s}^{2}}
$$

where $c_{1}$ and $c_{2}$ are dimensionless coefficients that are functions of the flexibility factor $d_{w}$, and are 
interrelated by

$$
c_{2}=c_{1} \frac{P_{s t} d_{w}}{\rho \ddot{X}_{g} H^{2}}
$$

These coefficients are plotted in Figure 4.8 and are also listed in Table 4.1. It is noteworthy that the ratio of these coefficients for the cantilever and top-supported walls range from 25.6 for rigid walls $\left(d_{w}=0\right)$ to 3.65 for walls with $d_{w}=40$. This large variation is due partly to the difference in the top support conditions and partly to the significantly different distributions of wall pressures in the two cases (see Figure 4.4).

For some insight into the magnitude of the maximum displacements that may be encountered in practice, consider a concrete wall of height $\mathrm{H}=15 \mathrm{ft}$ and thickness $t_{w}=1.5 \mathrm{ft}$ retaining a medium with $v_{s}=400 \mathrm{ft} / \mathrm{sec}$ and subjected to a ground motion with $\ddot{X}_{\mathrm{g}}=0.3 \mathrm{~g}$, where $g$ is the gravitational acceleration. With $E_{w}=3 \times 10^{6} \mathrm{psi}, v_{w}=0.17$, and a unit weight for the soil of $\gamma=\rho g=100 \mathrm{lb} / \mathrm{ft}^{3}$, $\mathrm{d}_{\mathrm{w}}$ is determined from equation (38) to be 13.4. This leads to $c_{2}=0.427$ for the cantilever wall and to $c_{2}=0.063$ for the top constrained wall. The corresponding maximum displacements, determined from equation (6), are $0.039 \%$ of the wall height for the cantilever wall and only $0.006 \%$ of the wall height for the top-constrained wall. Even with a dynamic amplification factor of 2.0 which, based on information presented in the following sections, represents a reasonable maximum for intense earthquake ground motions, these values are below the $0.1 \%$ to $0.4 \%$ range widely accepted as representing the displacement ratios required for the development of a limit state in the backfill material. ${ }^{21}$ 
Table 4.1: Normalized 'static' values of total wall force $P_{s t}$, centroidal height $h_{s t}$, base shear $\left(V_{b}\right)_{s t}$, base moment $\left(M_{b}\right)_{s t}$, and of displacement factors $c_{1}$ and $c_{2}$ for clamped-free (C-F) and clamped-hinged (C-H) walls.

\begin{tabular}{|c|c|c|c|c|c|c|c|c|c|c|}
\hline \multirow{3}{*}{$d_{w}$} & \multirow{2}{*}{\multicolumn{2}{|c|}{$\frac{P_{s t}}{\partial \ddot{X}_{g} H^{2}}$}} & \multirow{2}{*}{\multicolumn{2}{|c|}{$\frac{h_{s t}}{H}$}} & \multirow{3}{*}{$\frac{\frac{\left(V_{b}\right)_{s t}}{P_{s t}}}{\mathrm{C}-\mathrm{H}^{*}}$} & \multirow{3}{*}{$\frac{\frac{\left(M_{b}\right)_{s t}}{P_{s t} H}}{\mathrm{C}-\mathrm{H}^{*}}$} & \multicolumn{4}{|c|}{ Displacement Factors } \\
\hline & & & & & & & \multicolumn{2}{|c|}{$c_{1}$} & \multicolumn{2}{|c|}{$c_{2}$} \\
\hline & C-F & $\mathrm{C}-\mathrm{H}$ & C-F & $\mathrm{C}-\mathrm{H}$ & & & $C-F$ & $\mathrm{C}-\mathrm{H}$ & C-F & $\mathrm{C}-\mathrm{H}$ \\
\hline 0 & 0.940 & 0.940 & 0.599 & 0.599 & 0.528 & 0.127 & 0.1572 & 0.00614 & 0.0000 & 0.0000 \\
\hline 1 & 0.838 & 0.931 & 0.553 & 0.600 & 0.526 & 0.126 & 0.1394 & 0.00609 & 0.1169 & 0.0057 \\
\hline 2 & 0.770 & 0.922 & 0.517 & 0.601 & 0.524 & 0.125 & 0.1254 & 0.00604 & 0.1931 & 0.0111 \\
\hline 3 & 0.721 & 0.914 & 0.488 & 0.602 & 0.522 & 0.124 & 0.1140 & 0.00598 & 0.2465 & 0.0164 \\
\hline 4 & 0.683 & 0.906 & 0.463 & 0.604 & 0.520 & 0.123 & 0.1047 & 0.00593 & 0.2859 & 0.0215 \\
\hline 5 & 0.653 & 0.898 & 0.443 & 0.605 & 0.518 & 0.122 & 0.0968 & 0.00588 & 0.3161 & 0.0264 \\
\hline 10 & 0.561 & 0.864 & 0.375 & 0.610 & 0.508 & 0.118 & 0.0711 & 0.00564 & 0.3991 & 0.0487 \\
\hline 15 & 0.511 & 0.834 & 0.336 & 0.615 & 0.499 & 0.114 & 0.0568 & 0.00542 & 0.4355 & 0.0678 \\
\hline 20 & 0.477 & 0.808 & 0.310 & 0.620 & 0.491 & 0.110 & 0.0477 & 0.00521 & 0.4551 & 0.0842 \\
\hline 25 & 0.451 & 0.785 & 0.292 & 0.624 & 0.483 & 0.107 & 0.0414 & 0.00502 & 0.4669 & 0.0986 \\
\hline 30 & 0.431 & 0.765 & 0.279 & 0.628 & 0.476 & 0.104 & 0.0367 & 0.00485 & 0.4745 & 0.1113 \\
\hline 35 & 0.413 & 0.747 & 0.268 & 0.631 & 0.470 & 0.101 & 0.0331 & 0.00468 & 0.4796 & 0.1225 \\
\hline 40 & 0.399 & 0.731 & 0.259 & 0.635 & 0.464 & 0.099 & 0.0303 & 0.00453 & 0.4832 & 0.1325 \\
\hline
\end{tabular}

* For C-F walls, $\left(V_{b}\right)_{s t}=P_{s t}$ and $\left(M_{b}\right)_{s t}=P_{s t} h_{s t}$ 


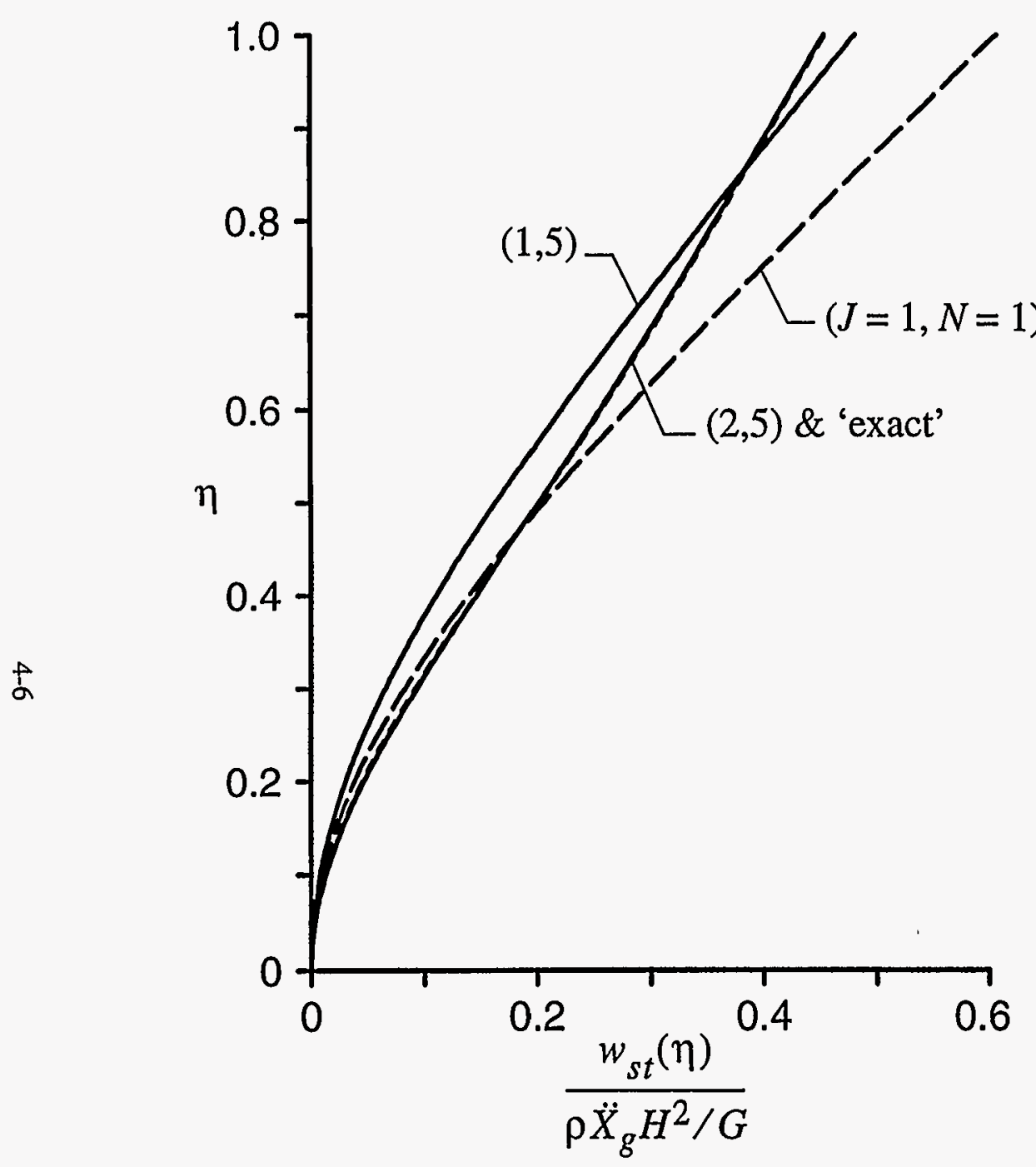

(a) Wall Displacements

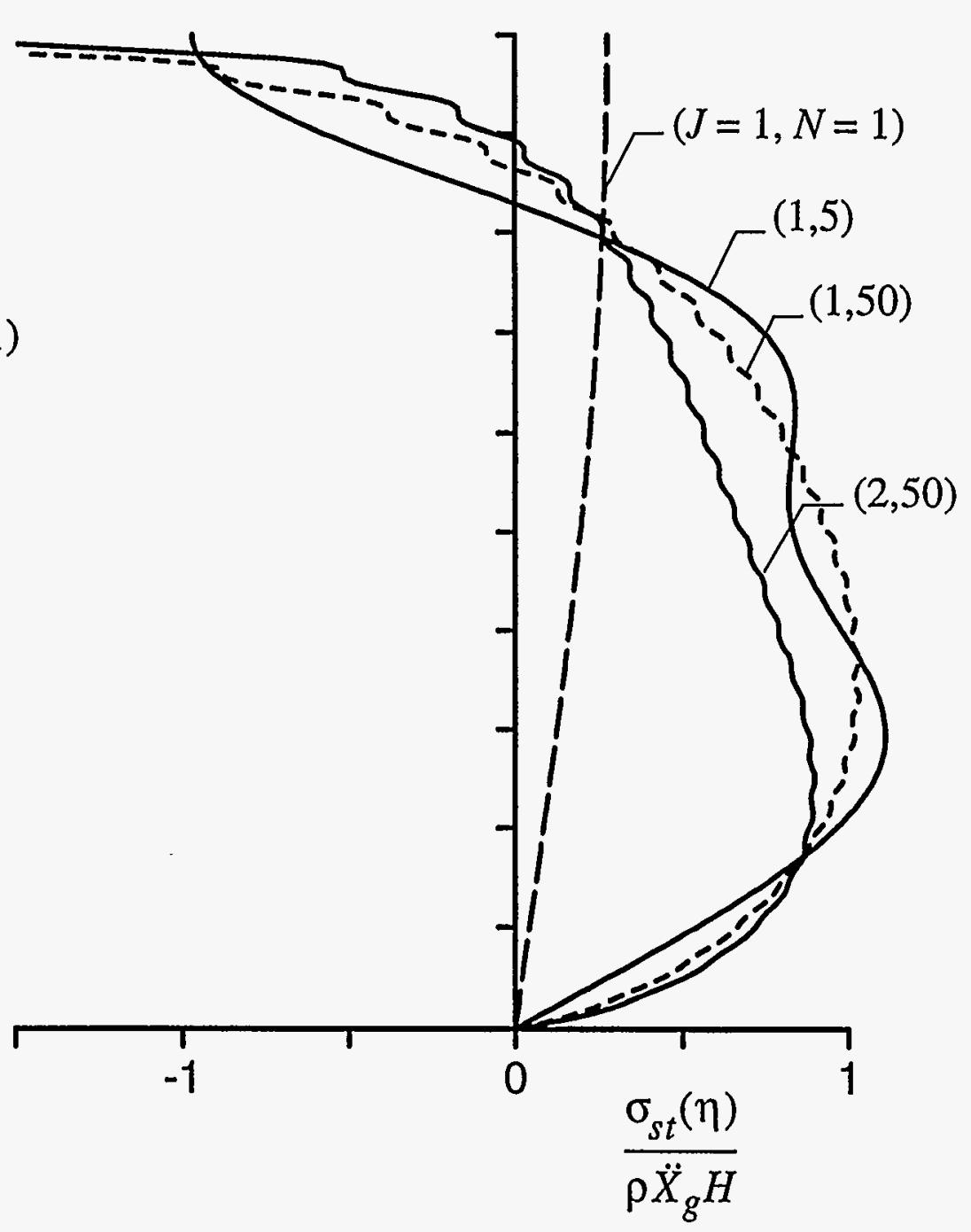

(b) Wall Pressures

Figure 4.1 Convergence of wall displacements and pressures for statically excited systems with C-F walls; $d_{w}=20, \mu_{w}=0, v=1 / 3$. 

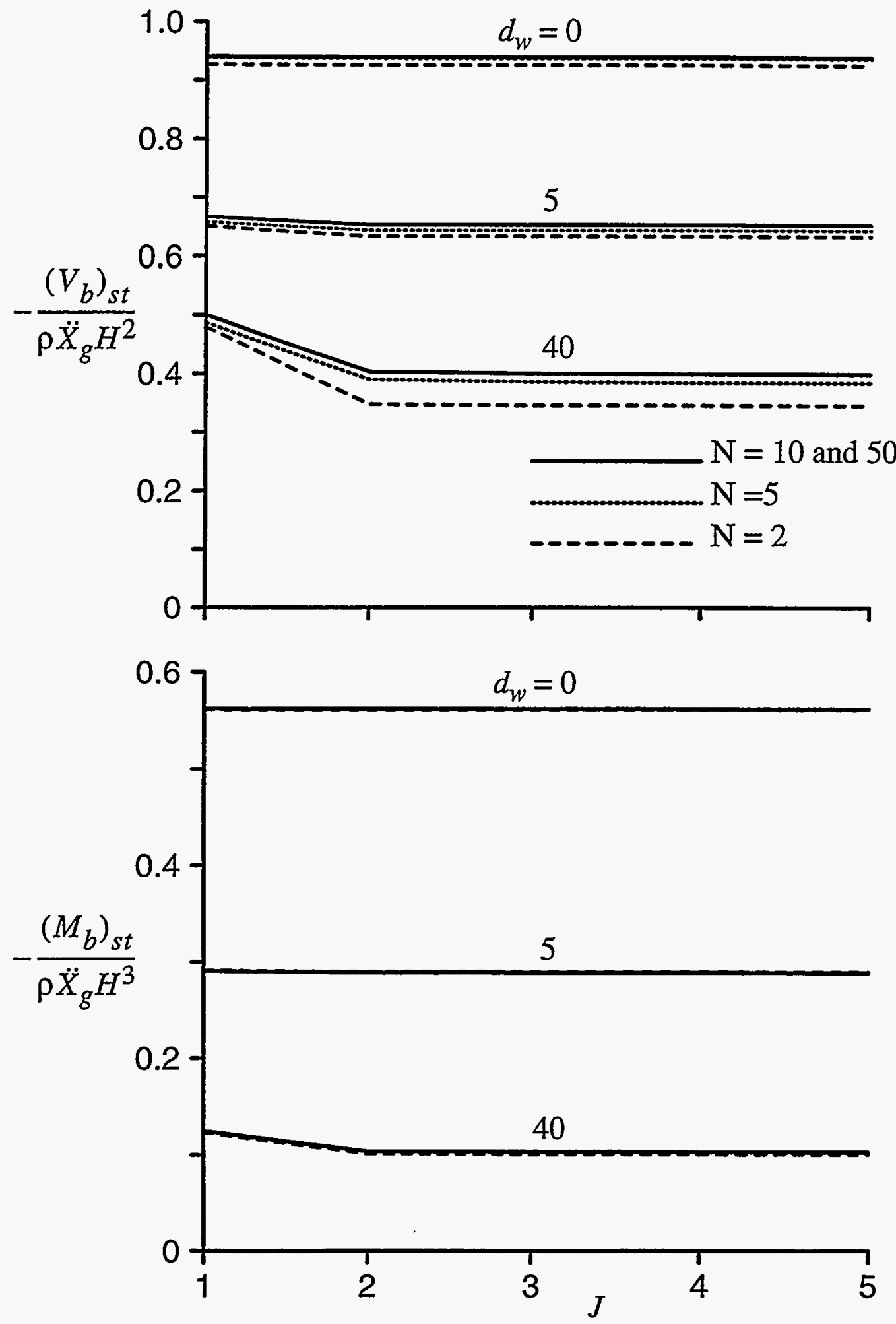

Figure 4.2 Convergence of base shear and base moment in wall of statically excited systems; C-F walls of different flexibilities, $\mu_{w}=0, v=1 / 3$. 

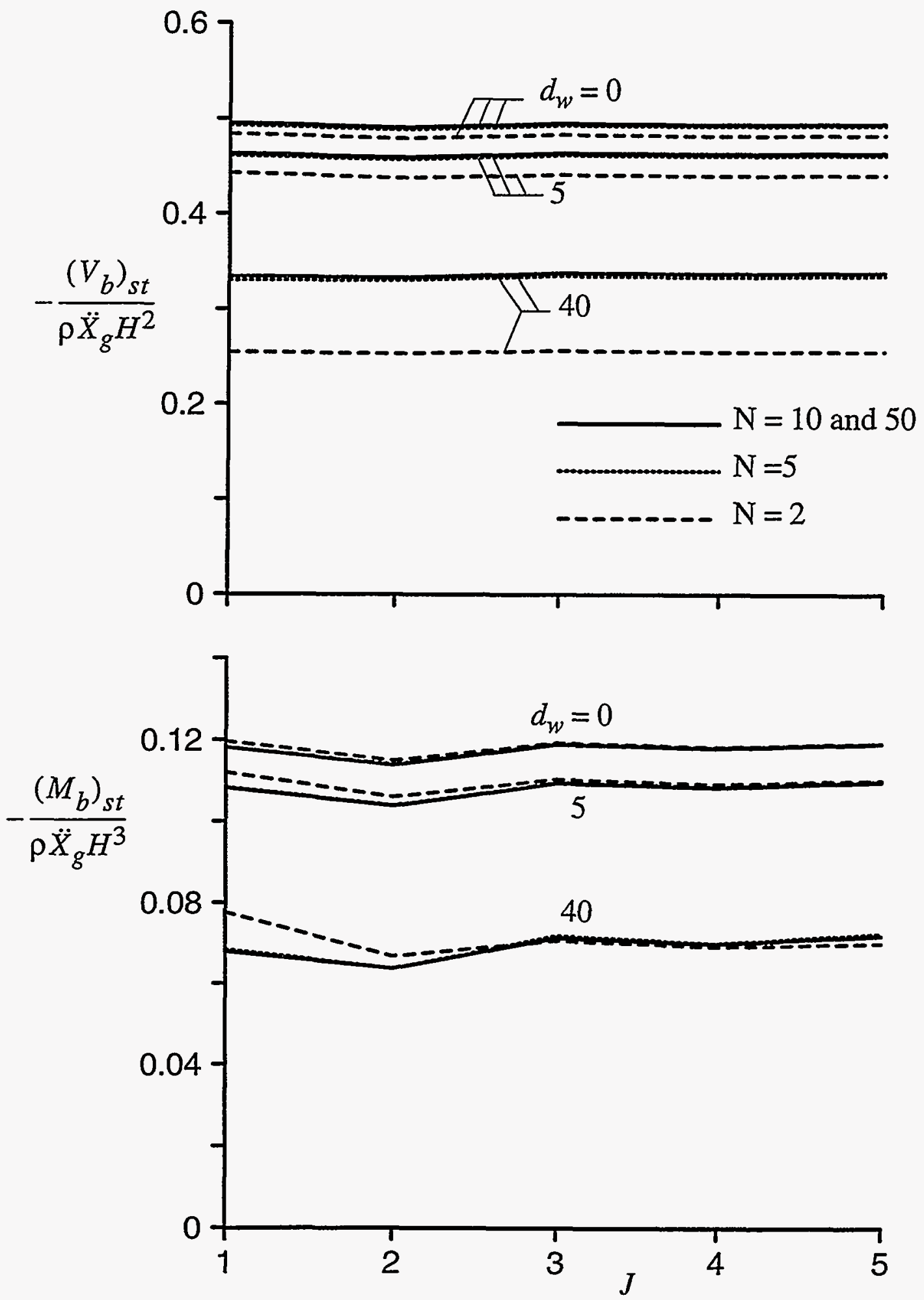

Figure 4.3 Convergence of base shear and base moment in wall of statically excited systems; $\mathrm{C}-\mathrm{H}$ walls of different flexibilities, $\mu_{\mathrm{w}}=0, v=1 / 3$. 


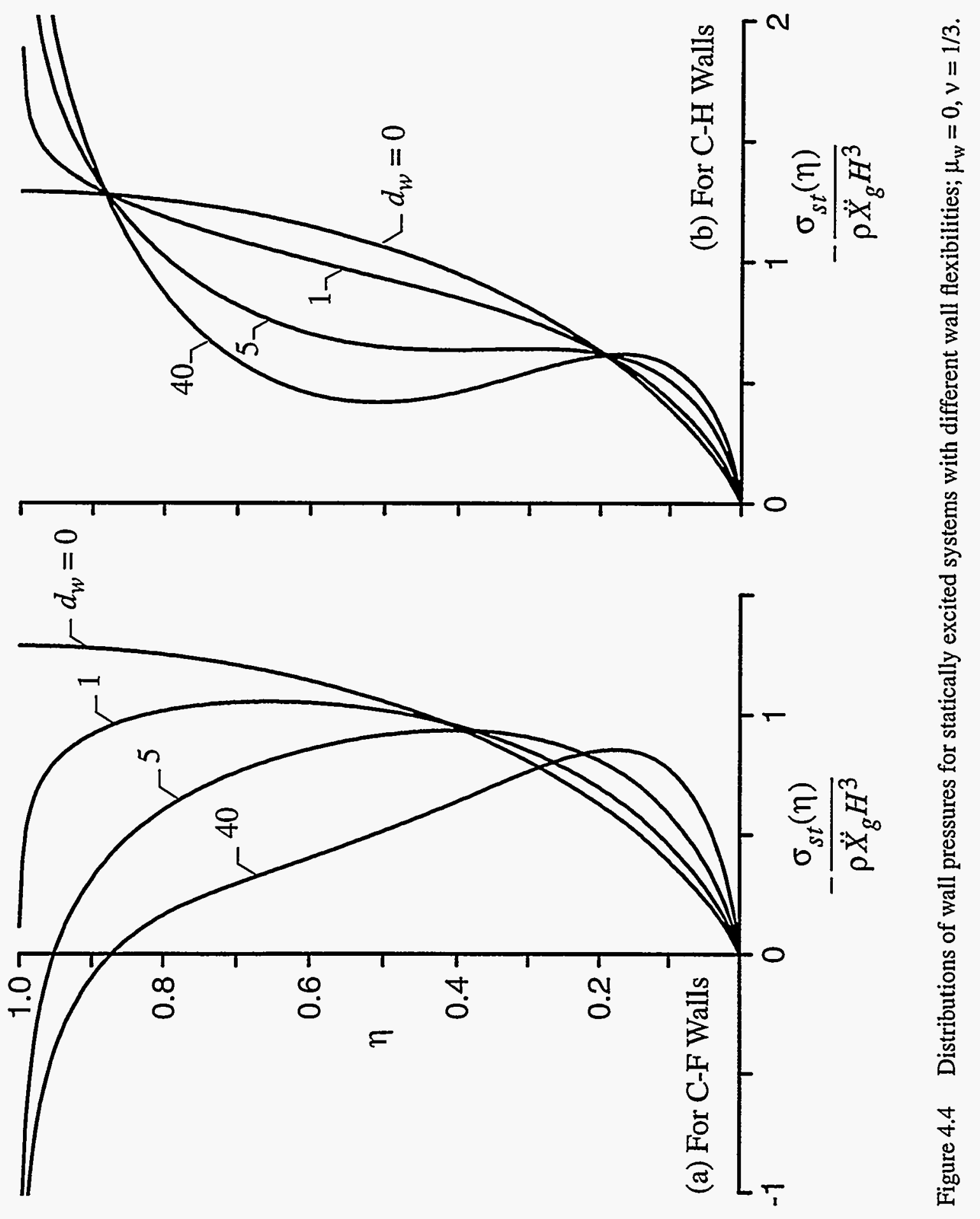



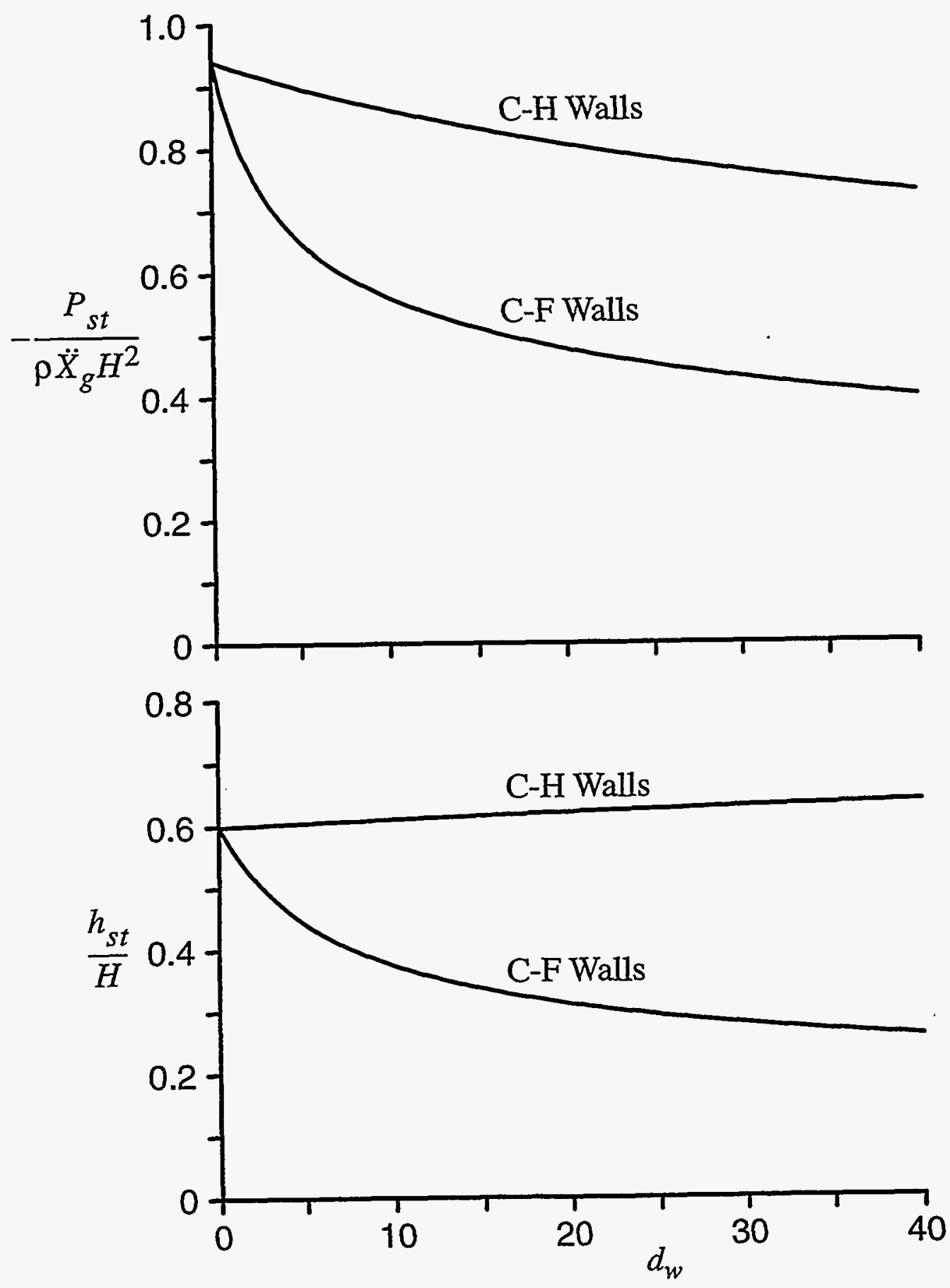

Figure 4.5 Normalized values of total force per unit of wall length and of centroidal height for statically excited systems with different wall flexibilities; $\mu_{w}=0, v=1 / 3$. 


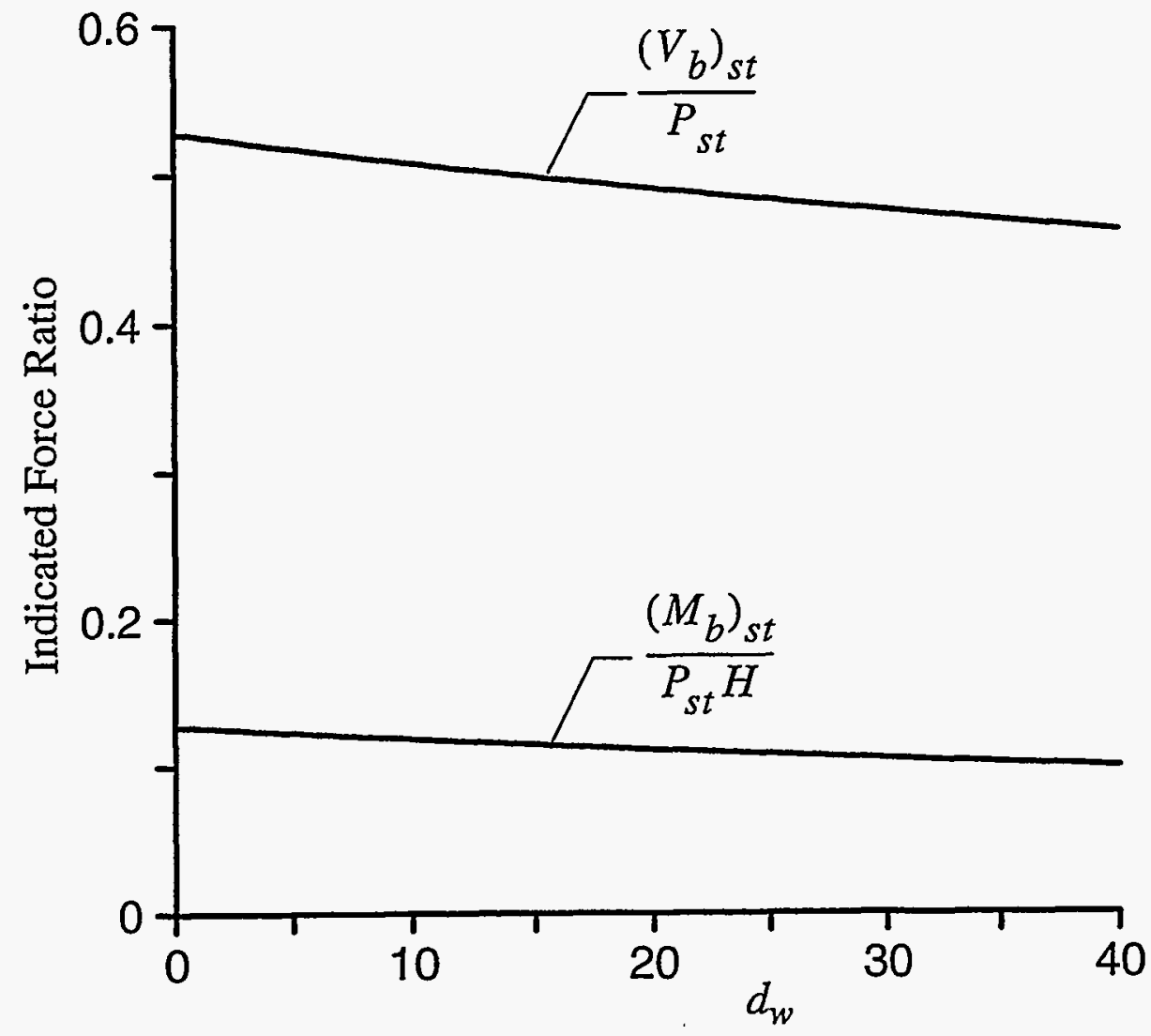

Figure 4.6 Normalized values of base shear and base moment per unit of wall length of statically excited systems; $C-H$ walls of different flexibilities, $\mu_{w}=0, v=1 / 3$. 


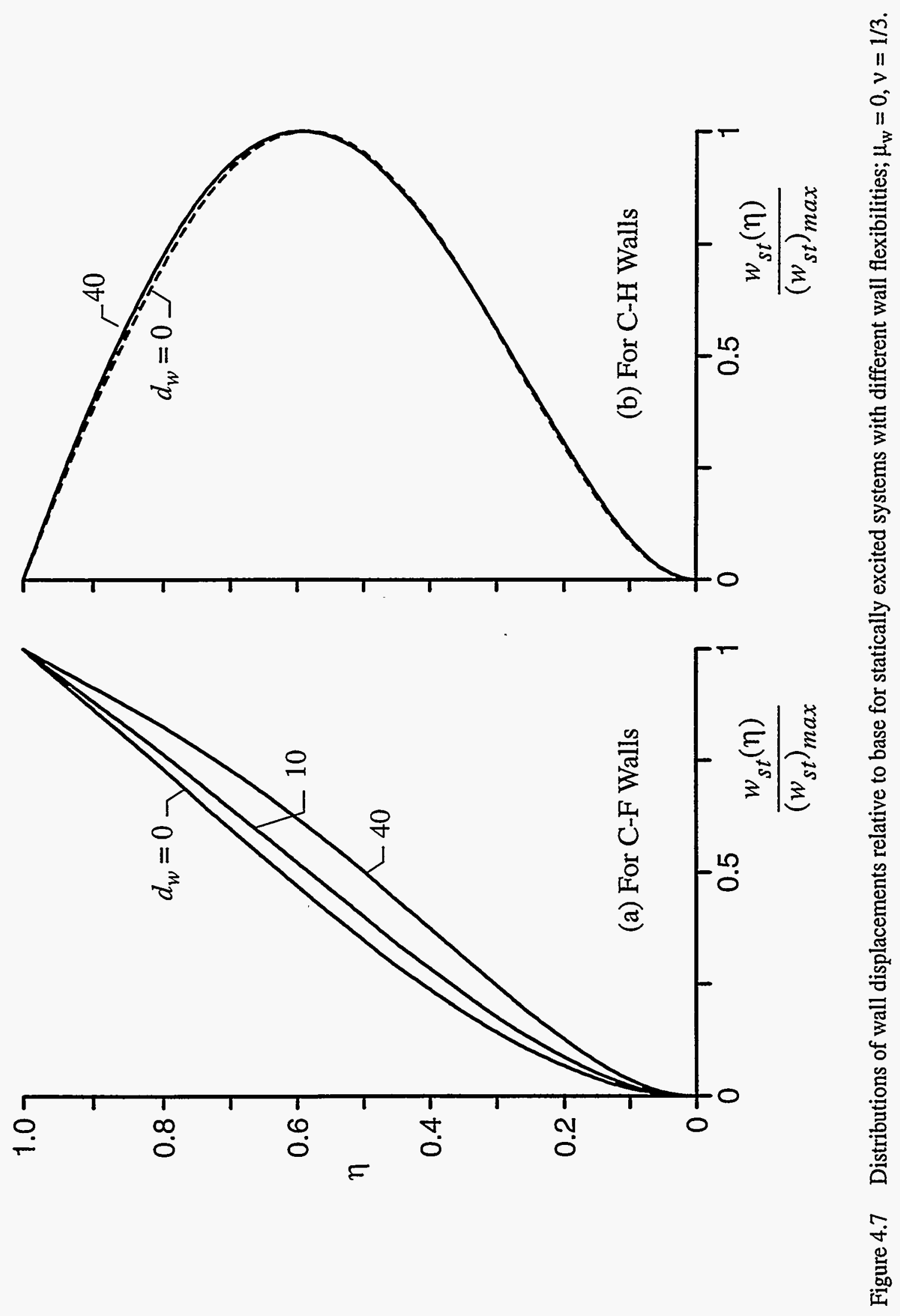




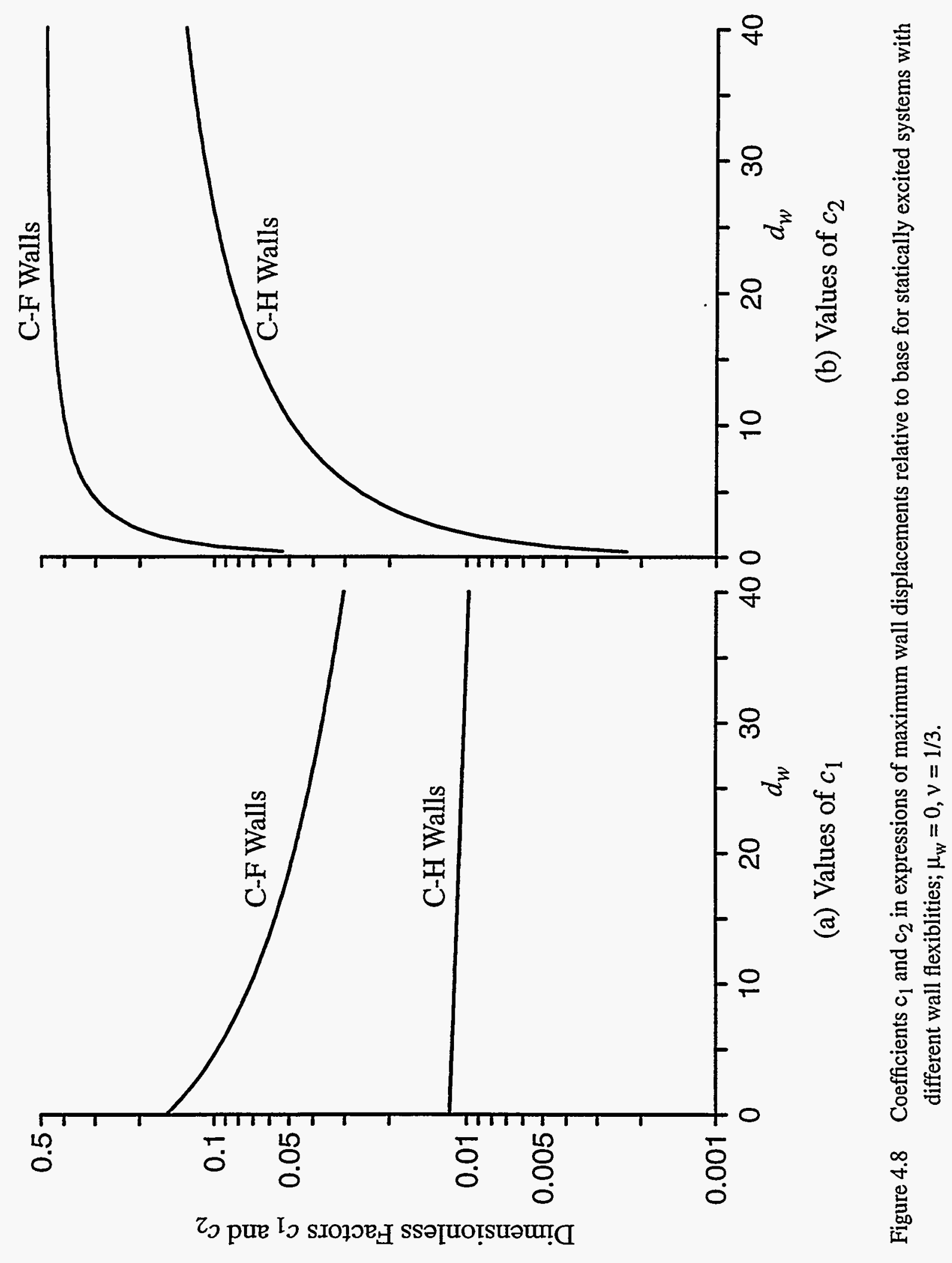




\section{SECTION 5}

\section{HARMONIC RESPONSE}

For the results presented so far, the dominant period of the excitation was considered to be long compared to the natural period of the retained material. In this section, the steady-state response of the system to a harmonic excitation of an arbitrary frequency is examined.

In the left part of Figure 5.1, the real-valued amplitude or maximum value of the total wall force per unit of length, $\mathrm{P}_{\max }$, for harmonically excited systems with a cantilever wall are plotted as a function of the frequency ratio $\omega / \omega_{1}$ for several values of the flexibility factor $d_{w}$. The results, which for the cantilever system considered also define the amplitude of the base shear per unit of wall length, $\left(V_{b}\right)_{\max }$, are normalized with respect to the common factor $\rho \ddot{X}_{\mathrm{g}} \mathrm{H}^{2}$. As before, Poisson's ratio and the damping factor for the retained medium are taken as $v=1 / 3$ and $\delta=0.1$, with the damping factor for the wall as $\delta_{w}=0.04$ (or $2 \%$ of critical damping).

As would be anticipated from the information for statically excited systems presented in the upper part of Figure 7, an increase in wall flexibility reduces the resulting wall force. However, the reduction is by no means uniform over the full range of frequencies. In particular, the reduction is substantially smaller at and near resonance than under static conditions of loading.

The interrelationship of the dynamic and 'static' forces may better be appreciated from the right-hand plots of Figure 5.1, in which the ratio of the maximum values of the two forces (the amplification factor, $A F$ ) is plotted as a function of the frequency ratio for the same three values of the flexibility factor $\mathrm{d}_{\mathrm{w}}$.

It is observed that: (a) the peak or resonant values of the amplification factors occur at exciting frequencies equal to the natural frequencies of the stratum, i.e., when $\omega / \omega_{1}=1,3,5, \ldots$; (b) the absolute maximum amplification factors are attained at the fundamental frequency of the stratum; and (c) the latter factors are quite sensitive to the relative flexibility factor $d_{w}$. For a rigid wall $\left(d_{w}=0\right)$, it is well known ${ }^{6,7}$ that the absolute maximum amplification factor is $1 / \sqrt{\delta}$, or 3.16 for the value of $\delta=0.1$ considered. By contrast, for flexible walls, this factor is larger due to the reduced capacity of such walls to reflect and dissipate by radiation the waves impinging on them. As $d_{w}$ tends to infinity, the soil-wall system tends to respond as an unconstrained cantilever shear-beam, and the absolute 
maximum amplification factor tends to $1 / \delta=10$, the value applicable to a viscously damped singledegree-of-freedom oscillator.

In Figure 5.2, the absolute maximum values of the amplification factors for cantilever and top-constrained walls are compared over the complete range of $d_{w}$ values examined. It is observed that the results for the top-constrained wall are significantly lower than for the cantilever wall. As previously indicated, this is due to the fact that, for a specified, finite value of the relative flexibility factor $d_{w}$, the effective stiffness of the top-constrained system is higher than that of the cantilever. 

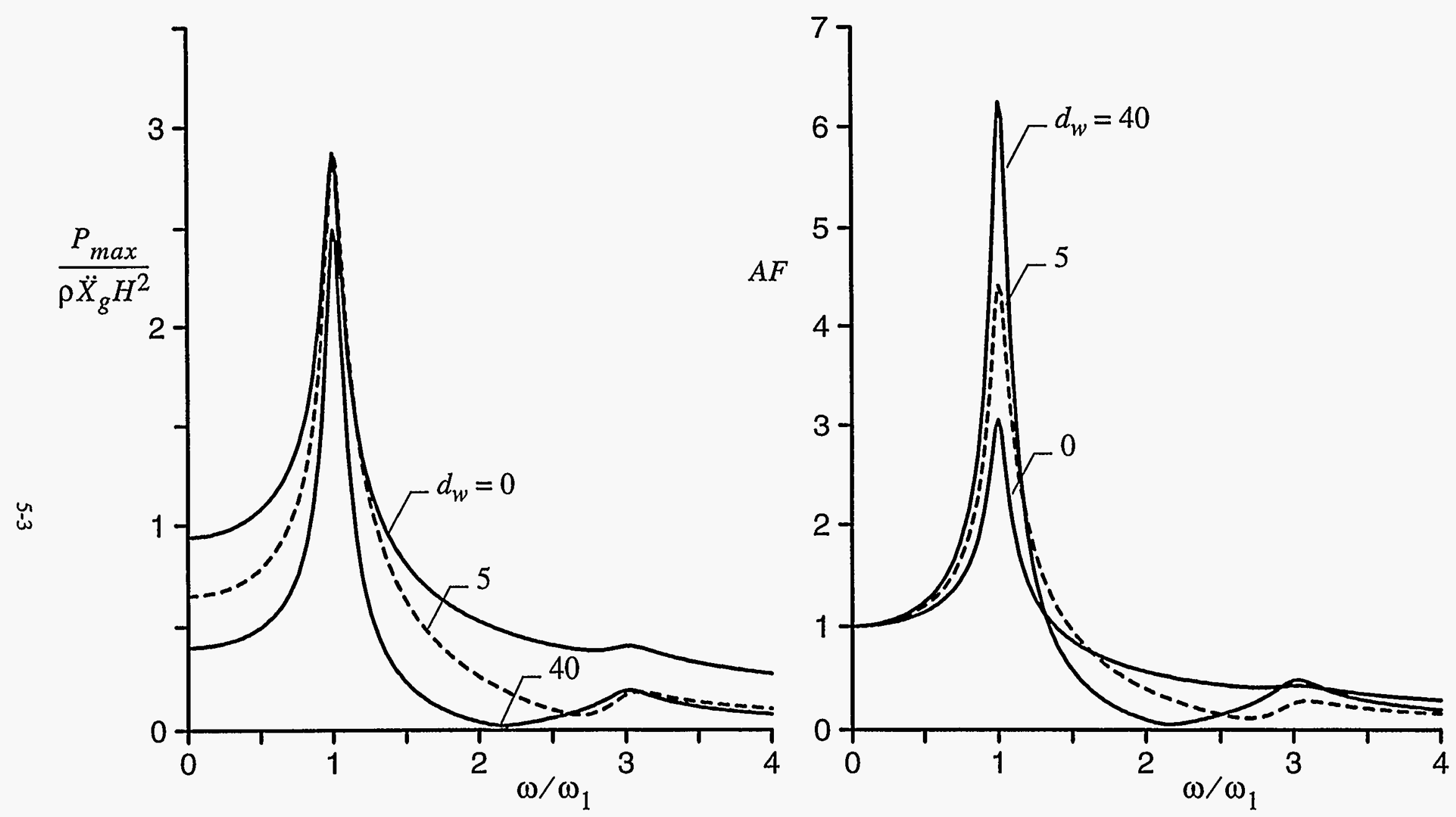

Figure 5.1 Frequency response curves for base shear per unit of wall length of harmonically excited systems; C-F walls of different flexibilities, $\mu_{w}=0, \delta_{w}=0.04, v=1 / 3, \delta=0.01$. 


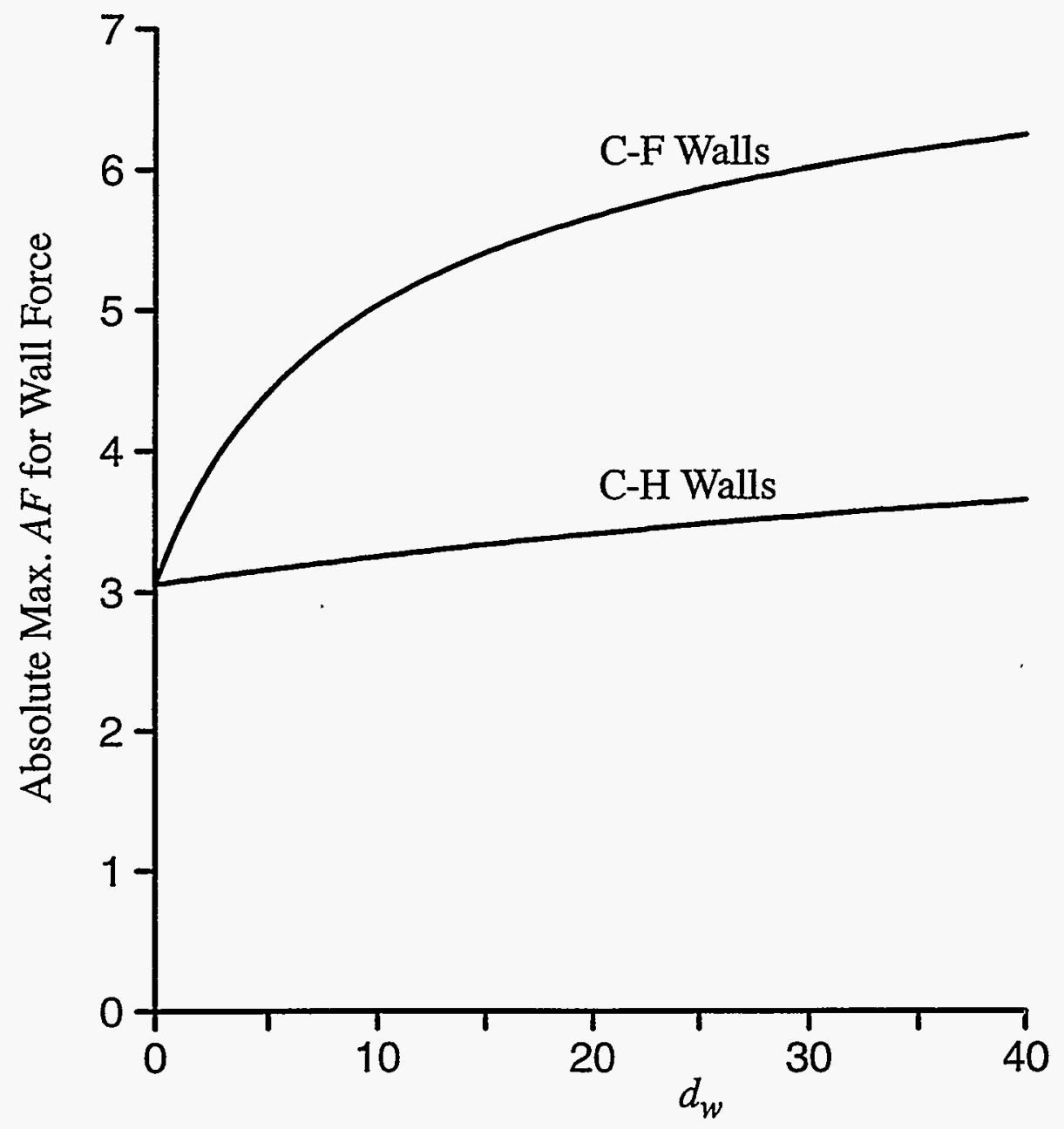

Figure 5.2 Maximum amplification factor for total force in wall of harmonically excited systems with different wall flexibilities; $\mu_{w}=0, \delta_{w}=0.04, v=1 / 3, \delta=0.01$. 


\section{SECTION 6}

\section{TRANSIENT RESPONSE}

Figure 6.1 shows normalized values of the absolute maximum wall force per unit of length, $\left|P_{\max }\right|$, for systems subjected to the first $6.3 \mathrm{sec}$ of the N-S component of the $1940 \mathrm{El}$ Centro, California earthquake record, the peak acceleration of which is $\ddot{X}_{g}=0.312 \mathrm{~g}$. The plots on the left are for cantilever walls, while those on the right are for top-constrained walls. The system parameters are the same as those for the harmonically excited systems examined in the preceding sections and are also identified on the figure heading. The results are plotted as a function of $T_{1}=2 \pi / \omega_{1}=4 \mathrm{H} / \mathrm{v}_{\mathrm{s}}$, the fundamental natural period of the soil stratum when it is considered to respond as an unconstrained cantilever shear-beam. As a measure of the range of $T_{1}$ values that may be encountered in practice, it is noted that for values of $\mathrm{v}_{s}$ between 250 and $1000 \mathrm{ft} / \mathrm{sec}$ and values of $H$ between 10 and $50 \mathrm{ft}$, the value of $T_{1}$ falls in the range of 0.04 to $0.8 \mathrm{sec}$.

The plots in Figure 6.1 are similar to, but by no means the same as, the response spectra for similarly excited, viscously damped single-degree-of-freedom systems. Specifically, for low-natural period, stiff strata, the wall force is the same as that obtained under static conditions of loading. With increasing $T_{1}$ or increasing flexibility of the stratum, the force levels increase, and after attaining nearly horizontal plateaus, they reach values that may well be less than the low-period, static values. For reasons already explained for statically and harmonically excited systems, the reduction in the force level achieved with a specified value of the relative wall flexibility factor $d_{w}$ is smaller for top-constrained walls than for cantilever walls.

The interrelationship of the maximum dynamic and long-period, static wall forces can better be seen in Figure 6.2, in which the information already displayed in Figure 6.1 is replotted in the form of amplification factors.

In Figure 6.3, the average values of the amplification factors for total wall force in the period range from $T_{1}=0.1$ to $0.5 \mathrm{sec}$ are replotted as a function of the flexibility factor $d_{w}$. The period range considered corresponds to the highly amplified, nearly horizontal region of the plots in Figures 6.1 and 6.2. It is observed that, for the cantilever walls, these factors range from 1.32 to 1.89 , whereas for the top-supported walls, they range from 1.32 to 1.51 . It should be recalled that these results are for a medium with a damping factor $\delta=0.1$ (5\% of critical damping). An increase in soil damping will 
naturally further reduce the amplification factors.

The normalized values of the centroidal heights $h$ for the seismically excited systems are finally plotted in Figure 6.4 as a function of the fundamental period of the stratum $T_{1}$. These heights, which for cantilever walls represent the heights by which the maximum wall force must be multiplied to yield the overturning base moment, are relatively insensitive to variations in $T_{1}$, and may, for all practical purposes, be taken equal to those reported in Figure 4.5 and Table 4.1 for the corresponding statically excited systems. The same can also be shown to be true of the normalized values of base shear and base moment, $\left(\mathrm{V}_{\mathrm{b}}\right)_{\max } / \mathrm{P}_{\max }$ and $\left(\mathrm{M}_{\mathrm{b}}\right)_{\max } / \mathrm{P}_{\max } \mathrm{H}$. 


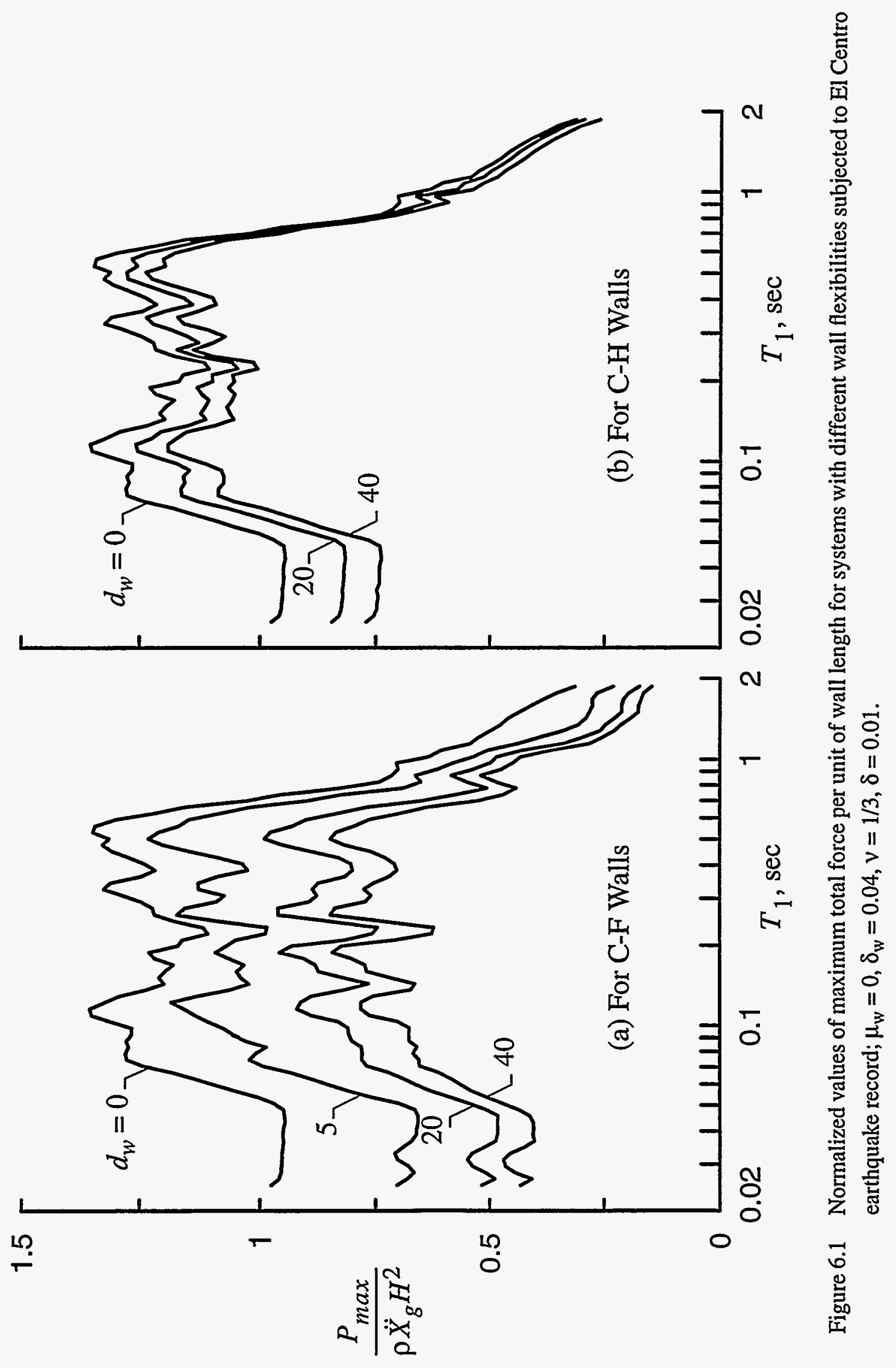




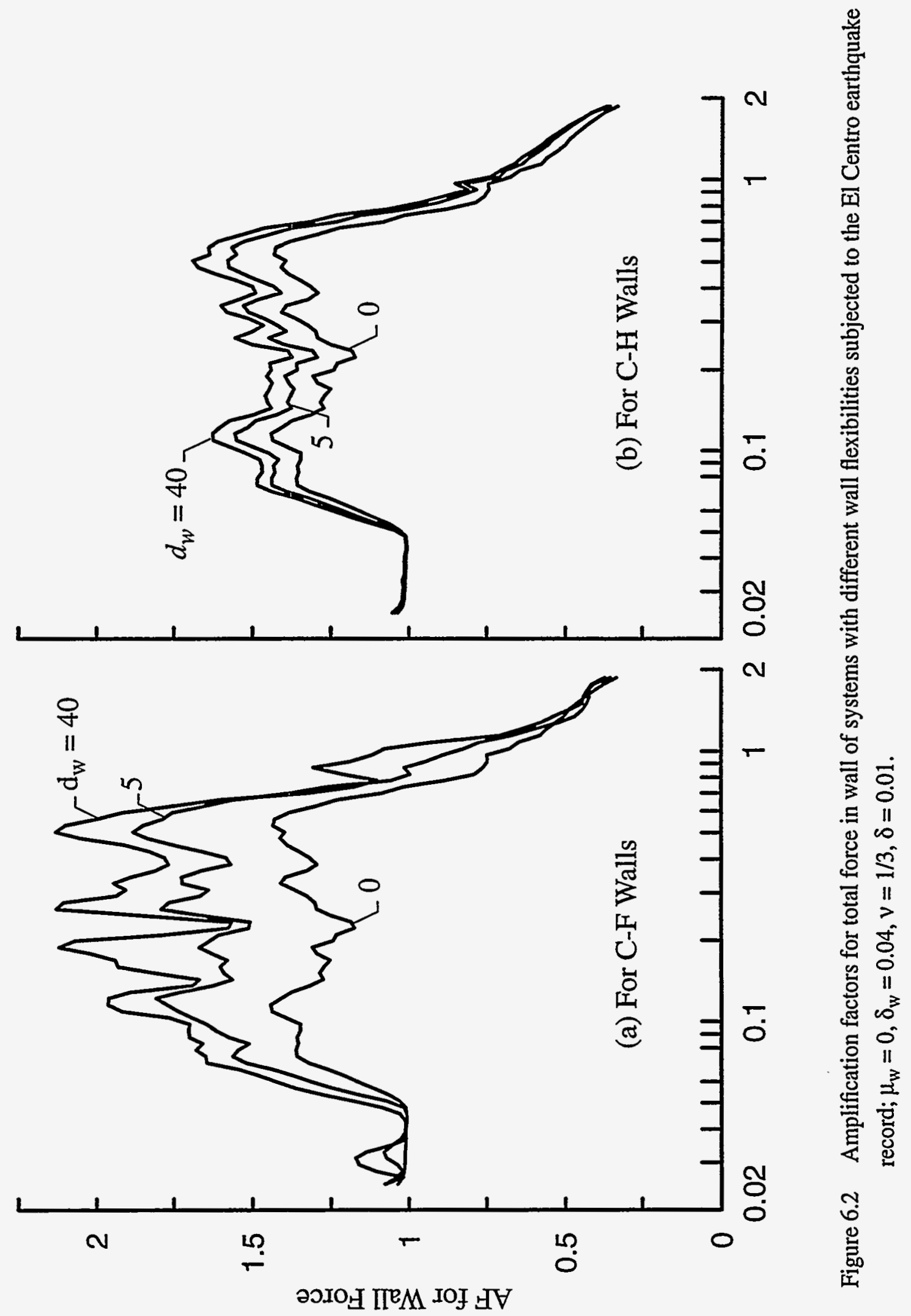




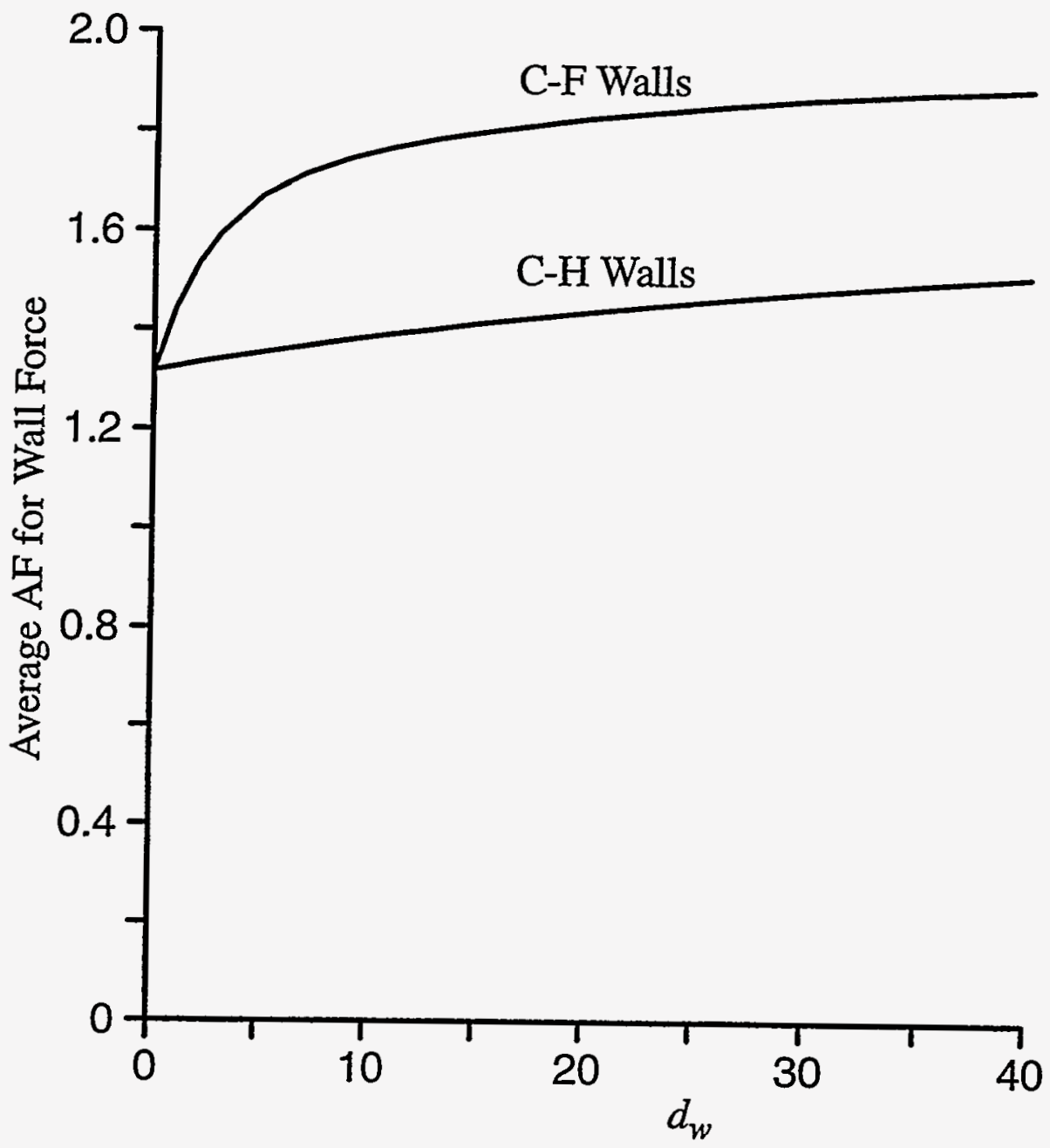

Figure 6.3 Average amplification factors for total wall force of systems subjected to El Centro earthquake record; $\mu_{w}=0, \delta_{w}=0.04, v=1 / 3, \delta=0.01 ; A F$ averaged over period range $T_{1}=0.1$ to $0.5 \mathrm{sec}$. 


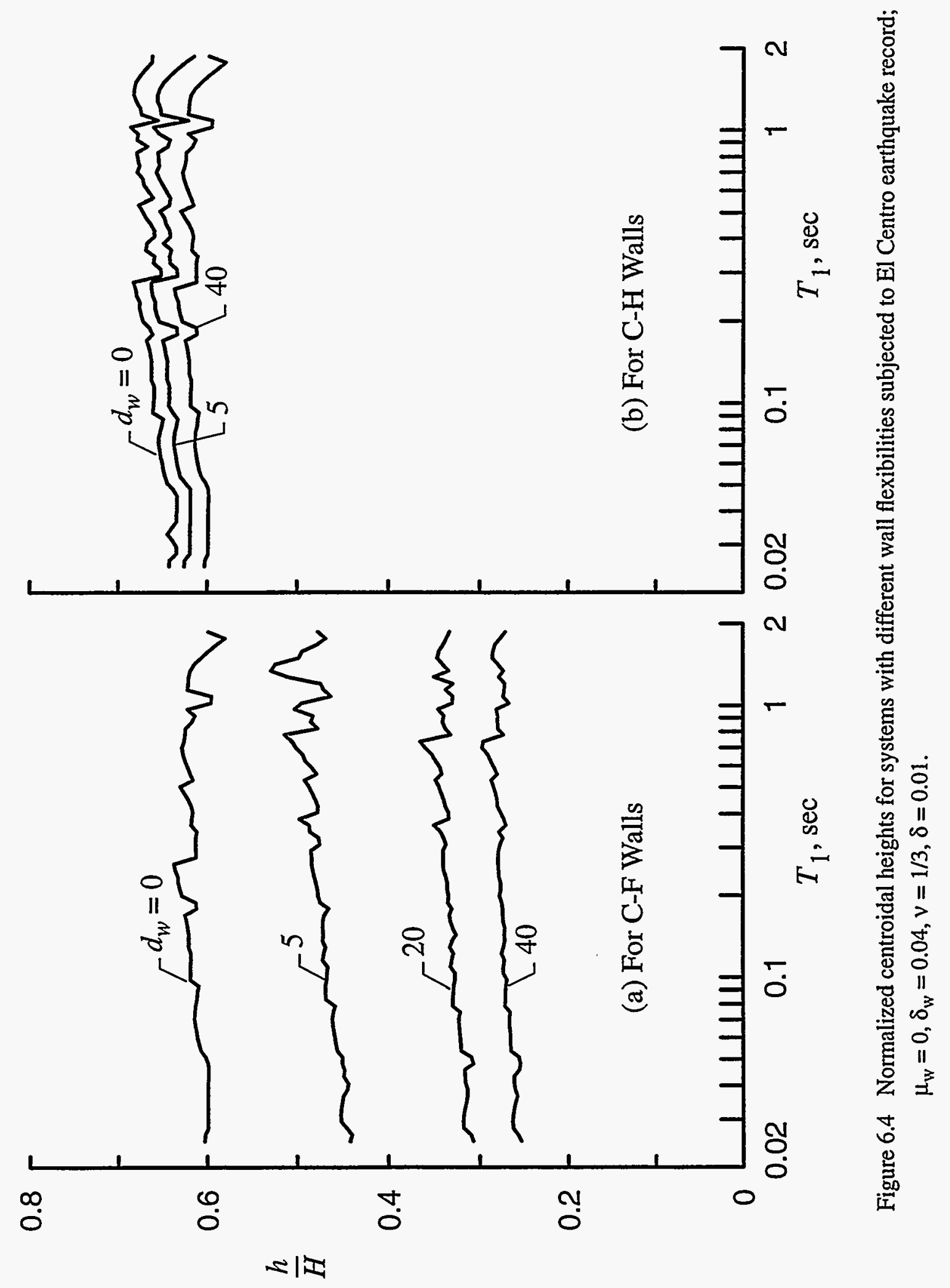




\section{SECTION 7}

\section{EFFECT OF WALL INERTIA}

For the systems considered so far, the wall mass was presumed to be negligible compared to the mass of the retained medium. The inertia of the wall has a twofold effect: (a) it modifies (generally decreases) the wall pressures induced by the retained medium; and (b) it induces additional forces on the wall. The net effect, which is generally an increase in the magnitude of the wall forces, may be evaluated exactly from the information presented, but the following simpler, approximate procedure would be adequate for all practical purposes.

The maximum force per unit of wall length for a wall with mass, $\mathrm{P}_{\max }^{\mu}$, may be related to that of the massless wall, $\mathrm{P}_{\max }$, by

$$
\mathrm{P}_{\max }^{\mu}=\mathrm{P}_{\max }+\mathrm{m}_{\mathrm{we}} \ddot{\mathrm{X}}_{\mathrm{g}}(\mathrm{AF})
$$

in which $\mathrm{m}_{\mathrm{we}}$ represents the effective mass per unit of wall length, and the amplification factor $A F$ may be taken equal to that for the massless wall. The value of $\mathrm{m}_{\mathrm{we}}$, normalized with respect to the corresponding wall mass $m_{w}=\mu_{w} H$, is plotted as a function of the relative flexibility factor $d_{w}$ in Figure 17. Both cantilever and top-constrained systems are considered. For rigid walls, the ratio is naturally unity, but for flexible walls, particularly for the more compliant cantilever systems, the effective mass is substantially smaller than the actual mass.

With the maximum force for a wall with mass determined, the corresponding base moment and end reactions may be determined by considering the latter forces to bear the same relationship to the wall force as those applicable to massless walls. This is tantamount to taking the centroidal height $h$ and the ratios of $\left(\mathrm{V}_{\mathrm{b}}\right)_{\max } / \mathrm{P}_{\max }$ and $\left(\mathrm{M}_{\mathrm{b}}\right)_{\max } / \mathrm{P}_{\max } \mathrm{H}$ for the wall with mass equal to those for a massless wall. 


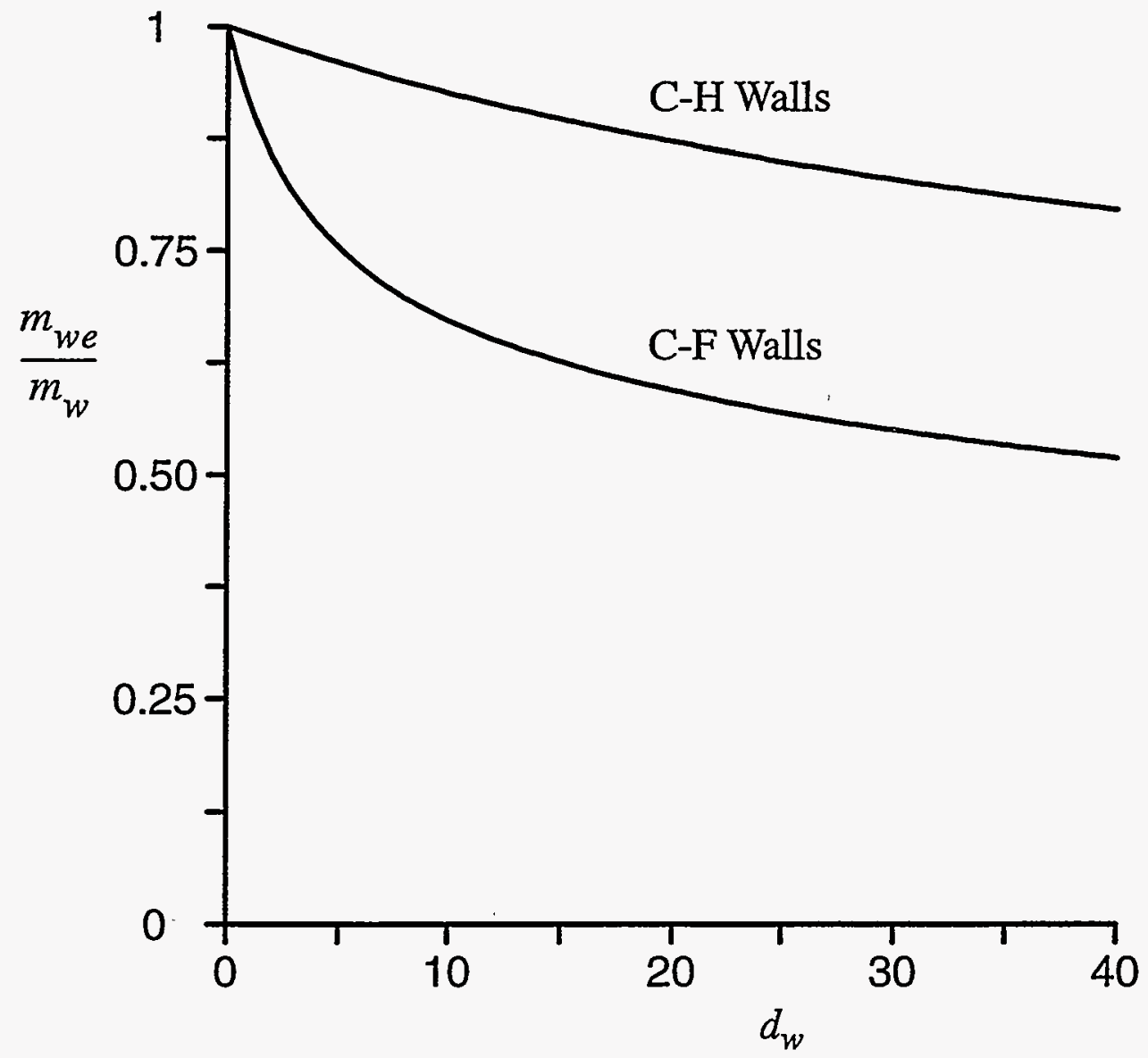

Figure 7.1 Effective wall mass for statically excited systems with different wall flexibilities; $v=1 / 3$. 


\section{SECTION 8}

\section{CONCLUSIONS}

Following are some of the more important conclusions of this study.

1. With the method of analysis and the numerical data presented, the dynamic response of the class of flexible retaining walls examined may be evaluated readily and with high degree of accuracy. The method, which makes use of Lagrange's equations of motion in combination with a recently proposed model for the action of soil-wall systems, is expected to prove of value in the analysis of a number of other problems as well.

2. The magnitudes and distributions of the wall displacements, wall pressures and associated forces induced by horizontal ground shaking in the systems examined are quite sensitive to the flexibility of the wall. Increasing this flexibility reduces the horizontal extensional stiffness of the retained medium relative to its shearing stiffness, and this reduction decreases the proportion of the soil inertia forces that gets transferred to the wall and, hence, the forces developed in it.

3. For realistic wall flexibilities, the total wall force or base shear for cantilever walls may well be less than one-half of that obtained for fixed-based, rigid walls, with the reduction in the base moment being even larger. Because of the greater effective stiffness of top-supported walls, the corresponding reductions for such walls are significantly smaller than for the cantilever systems.

4. Even for the $1940 \mathrm{El} \mathrm{Centro} \mathrm{earthquake} \mathrm{ground} \mathrm{motion} \mathrm{record,} \mathrm{the} \mathrm{maximum} \mathrm{wall} \mathrm{displacement}$ relative to the moving base for cantilever walls of realistic flexibilities is found to be less than the values of 0.1 to 0.4 percent of the wall height normally accepted as the minimum required to develop a limit state in the backfill material.

5. The comprehensive numerical solutions presented and their analysis provide not only valuable insights into the effects and relative importance of the numerous factors that influence the response of the systems examined, but also a sound framework for assessing the behavior of even more complex soil-wall systems. 


\section{SECTION 9}

\section{REFERENCES}

1. N. Mononobe and H. Matuo, 'On the determination of earth pressures during earthquakes', Proc. world engrg. congress, Tokyo, Japan, 1929, Vol. 9, Paper No. 388.

2. S. Okabe, 'General theory of earth pressure and seismic stability of retaining wall and dam', $J$. japan soc. civil engrs., 12 (1924).

3. H. B. Seed and R. V. Whitman, 'Design of earth retaining structures for dynamic loads', ASCE speciality conf. on lateral stresses in the ground and design of earth retaining structures, 1970 , pp. 103-147.

4. H. Matuo and S. Ohara, 'Lateral earth pressure and stability of quay walls during earthquakes', Proc. 2nd world conf. earthquake engrg., Tokyo, Japan, 1960.

5. J. H. Wood, 'Earthquake-induced soil pressures on structures', Report EERL 73-05, Earthquake Engineering Research Laboratory, California Institute of Technology, 1973.

6. A. Arias, F. J. Sanchez-Sesma and E. Ovando-Shelley, 'A simpiified elastic model for seismic analysis of earth-retaining structures with limited displacements', Proc. int. conf. on recent adv. in geotech. earthquake engrg. and soil dyn., St. Louis, MO, 1981, Vol. I, pp. 635-642.

7. A. S. Veletsos and A.H. Younan, "Dynamic soil pressures on rigid retaining walls," Earthquake engrg. \& struct. dyn.23, 275-301 (1994); see also Reply by Authors to Y. W. Chang and to J. P. Wolf, Earthquake engrg. \& struct. dyn. 24, 1285-1286 and 1293 (1995).

8. W. D. Liam Finn, M. Yogendrakumar, H. Otsu and R. S. Steedman, 'Seismic response of a cantilever retaining wall: centrifuge model test and dynamic analysis', Proc. fourth int. conf. on soil dyn. and earthquake engrg., Computational Mechanics Publications, Southampton, 1989, pp. 331-431.

9. K. Sun and G. Lin, 'Dynamic response of soil pressure on retaining wall', in Proc. Third Int. Conf. on Recent Advances in Geotech. Earthquake Engrg. and Soil Dyn., St. Louis, MO, 1995, Vol. I, pp. 347-350.

10. H. N. Nazarian and A. H. Hadjian, 'Earthquake-induced lateral soil pressures on structures', J. of the geotech. engrg. div. ASCE 105, 1049-1066 (1979).

11. S. Prakash, 'Analysis of rigid retaining walls during earthquakes', Proc. int. conf. on recent adv. in geotech. earthquake engrg. and soil dyn., St. Louis, MO, 1981, Vol. III, pp. 1-28.

12. R. V. Whitman, 'Seismic design of earth retaining structures', Proc. second int. conf. on recent adv. in geotech. earthquake engrg. and soil dyn., St. Louis, MO, 1991, Vol. II, pp. 1767-1777. 
13. A. S. Veletsos and A. H. Younan, 'Dynamic soil pressures on vertical walls', Proc. Third int. conf. on recent adv. in geotech. earthquake engrg. and soil dyn., St. Louis, MO, 1995, Vol III, pp. 1589-1604.

14. A. S. Veletsos and A. H. Younan, 'Dynamic modeling and response of soil-wall systems', J. of geotech. engrg. ASCE 120, 2155-2179 (1994).

15. A. S. Veletsos and B. Verbic, 'Vibration of viscoelastic foundations', Earthquake engrg. \& struct. dyn. 2, 87-102 (1973).

16. A. S. Veletsos and D. D. Nair, 'Seismic interaction of structures on hysteretic foundations', $J$. struct. div. ASCE 101, 109-129 (1975).

17. J. M. Roesset, R. V. Whitman and R. Dobry, 'Modal analysis for structures with foundation interaction', J. struct. div. ASCE 99, 399-416 (1973).

18. A. Pais and E. Kausel, 'Approximate formulas for dynamic stiffnesses of rigid foundations', Soil dyn. \& earthquake engrg. 7, 213-227 (1988).

19. R. W. Clough and J. Penzien, Dynamics of Structures, McGraw Hill, NY, 1994, pp. 344-351.

20. I. Stakgold, Green's Functions and Boundary Value Problems, John Wiley \& Sons, NY, 1979, pp. 280-281.

21. G. W. Clough and J. M. Duncan, 'Earth pressures', Foundation Engineering Handbook, Ed. by H. Y. Fang, Chapman \& Hall, NY, 1990, pp. 223-235.

22. D. Young and R. P. Felgar, 'Tables of characteristic functions representing normal modes of vibration of a beam', Engineering research series No. 44, Bureau of Engineering Research, The University of Texas, Austin, TX, 1949.

23. R. P. Felgar, 'Formulas for integrals containing characteristic functions of a vibrating beam', Circular No. 14, Bureau of Engineering Research, The University of Texas, Austin, TX, 1950. 


\section{SECTION 10}

\section{APPENDIX. NATURAL MODES AND INNER PRODUCTSS}

The jth natural mode of vibration of the beam or wall without the retained medium is given by

$$
\phi_{j}(\eta)=\cosh \lambda_{j} \eta-\cos \lambda_{j} \eta-\alpha_{j}\left[\sinh \lambda_{j} \eta-\sin \lambda_{j} \eta\right]
$$

in which $\lambda_{j}$ and $\alpha_{j}$ are dimensionless factors that depend on the end support conditions. The $j$ th circular natural frequency of the wall, $\omega_{w, j}$, is related to $\lambda_{j}$ by

$$
\omega_{w, j}=\frac{\lambda_{j}^{2}}{H^{2}} \sqrt{\frac{D_{w}}{\mu_{w}}}
$$

The first five values of $\lambda_{j}$ and $\alpha_{j}$ for the two sets of boundary conditions considered here are given in Young and Felgar ${ }^{22}$ and are reproduced in Table 11.1.

The inner products or integrals in the various expressions presented in the body of this paper are as follows:

$$
\begin{gathered}
\left\langle\psi_{\mathrm{n}}, \Psi_{\mathrm{n}}\right\rangle=0.5 \\
\left\langle\phi_{\mathrm{j}}, \phi_{\mathrm{j}}\right\rangle=1 \\
\left\langle\phi_{\mathrm{j}}^{\prime \prime}, \phi_{\mathrm{j}}^{\prime \prime}\right\rangle=\lambda_{\mathrm{j}}^{4} \\
\left\langle\phi_{\mathrm{j}}, 1\right\rangle=\left\{\begin{array}{c}
\mathrm{C}-\mathrm{F}: \quad \frac{2 \alpha_{\mathrm{j}}}{\lambda_{\mathrm{j}}} \\
\mathrm{C}-\mathrm{H}: \frac{2 \alpha_{\mathrm{j}}+(-1)^{\mathrm{j}+1} \sqrt{\alpha_{\mathrm{j}}^{2}+1}-\sqrt{\alpha_{\mathrm{j}}^{2}-1}}{\lambda_{\mathrm{j}}}
\end{array}\right.
\end{gathered}
$$




$$
\begin{aligned}
& \left\langle\phi_{j}, \eta\right\rangle=\left\{\begin{array}{l}
\text { C-F: } \frac{2}{\lambda_{j}^{2}} \\
C-H: \frac{2-\lambda_{j}\left[(-1)^{j} \sqrt{\alpha_{j}^{2}+1}+\sqrt{\alpha_{j}^{2}-1}\right]}{\lambda_{j}^{2}}
\end{array}\right. \\
& \left\langle\phi_{j}, \Psi_{n}\right\rangle= \begin{cases}\text { C-F: } & \frac{2 \lambda_{j} \varepsilon_{n}\left[\lambda_{j}-(-1)^{n+j} \alpha_{j} \varepsilon_{n}\right]}{\lambda_{j}^{4}-\varepsilon_{n}^{4}} \\
C-H: & \frac{1}{\lambda_{j}^{4}-\varepsilon_{n}^{4}}\left[\begin{array}{c}
2 \lambda_{j}^{2} \varepsilon_{n}+(-1)^{n-1} \lambda_{j}^{3}\left[(-1)^{j+1} \sqrt{\alpha_{j}^{2}+1}-\sqrt{\alpha_{j}^{2}-1}\right] \\
-(-1)^{n-1} \lambda_{j} \varepsilon_{n}^{2}\left[(-1)^{j} \sqrt{\alpha_{j}^{2}+1}-\sqrt{\alpha_{j}^{2}-1}\right]
\end{array}\right]\end{cases}
\end{aligned}
$$

in which $\varepsilon_{\mathrm{n}}=(2 \mathrm{n}-1) \pi / 2$. Equations (46)-(49) are given in Felgar ${ }^{23}$, while the rest were derived from basic principles. 
Table 10.1 Dimensionless factors $\lambda_{j}$ and $\alpha_{j}$ in expressions for natural modes of vibration of clamped-free (C-F) and clamped-hinged (C-H) flexural beams

\begin{tabular}{|c|c|c|c|c|}
\hline \multirow{2}{*}{$\begin{array}{c}\text { Order of } \\
\text { Mode } j\end{array}$} & \multicolumn{2}{|c|}{ C-F Beams } & \multicolumn{2}{c|}{ C-H Beams } \\
\cline { 2 - 5 } & $\lambda_{j}$ & $\alpha_{j}$ & $\lambda_{j}$ & $\alpha_{j}$ \\
\hline \hline 1 & 1.87510410 & 0.73409550 & 3.92660230 & 1.00077730 \\
2 & 4.69409113 & 1.01846644 & 7.06858275 & 1.00000144 \\
3 & 7.85475743 & 0.99922450 & 10.21017613 & 1.00000000 \\
4 & 10.99554074 & 1.00003355 & 13.35176878 & 1.00000000 \\
5 & 14.13716839 & 0.99999855 & 16.49336143 & 1.00000000 \\
\hline
\end{tabular}




\section{SECTION 11}

\section{NOTATION}

The following symbols are used in this report:

$a_{j} \quad$ elements of $\{A\}$

$\{A\}$ vector of normalized exciting forces

$c_{1}, c_{2}$ dimensionless factors in expressions for maximum wall displacement

$\mathrm{d}_{\mathrm{w}} \quad$ relative wall flexibility factor defined by equation (36)

$\mathrm{D}_{\mathrm{w}} \quad$ flexural rigidity per unit of wall length, defined by equation (5)

$D_{w}^{*} \quad$ complex-valued flexural rigidity of wall $\left[=D_{w}\left(1+i \delta_{w}\right)\right]$

$E_{w} \quad$ Young's modulus of elasticity for wall

$\mathrm{f}_{1} \quad$ fundamental cyclic frequency of retained medium when it is assumed to respond as an unconstrained cantilever shear-beam

$F_{j} \quad$ generalized force

$\mathrm{g}$ acceleration due to gravity

G shear modulus of elasticity for retained material

$\mathrm{G}^{*} \quad$ complex-valued shear modulus for retained material $[=\mathrm{G}(1+\mathrm{i} \delta)]$

$\mathrm{h}$ centroidal height, defined as distance from base to point of application for dynamic wall force $P$

i $\quad[=\sqrt{-1}]$

$\mathrm{j} \quad$ integer defining order of beam mode under consideration

$\mathrm{J} \quad$ integer defining total number of beam modes considered

$\mathrm{K}_{\mathrm{n}} \quad$ dynamic impedance of spring-dashpot combination when both the wall and the retained medium at the far-field vibrate in the $n$th natural mode of a uniform, cantilever shear-beam

$\mathrm{m}_{\mathrm{jj}} \quad$ elements of $[\mathrm{M}]$

$\mathrm{m}_{\mathrm{w}} \quad$ mass per unit length of wall

$m_{w e} \quad$ effective mass per unit length of wall

[M] mass matrix

$\tilde{M} \quad$ instantaneous value of overturning base moment per unit of wall length induced by force $P$

$\mathrm{M}_{\mathrm{b}} \quad$ bending moment at base of wall

$\mathrm{n} \quad$ integer defining order of shear-beam mode under consideration

$\mathrm{N}$ integer defining total number of shear-beam modes considered

$P \quad$ instantaneous value of total dynamic force per unit of wall length

$\mathrm{q}_{\mathrm{j}} \quad$ generalized coordinate

$\mathrm{Q}_{\mathrm{j}} \quad$ amplitude of $j$ th generalized coordinate

$\{\mathrm{Q}\} \quad$ vector of amplitudes of generalized coordinates 


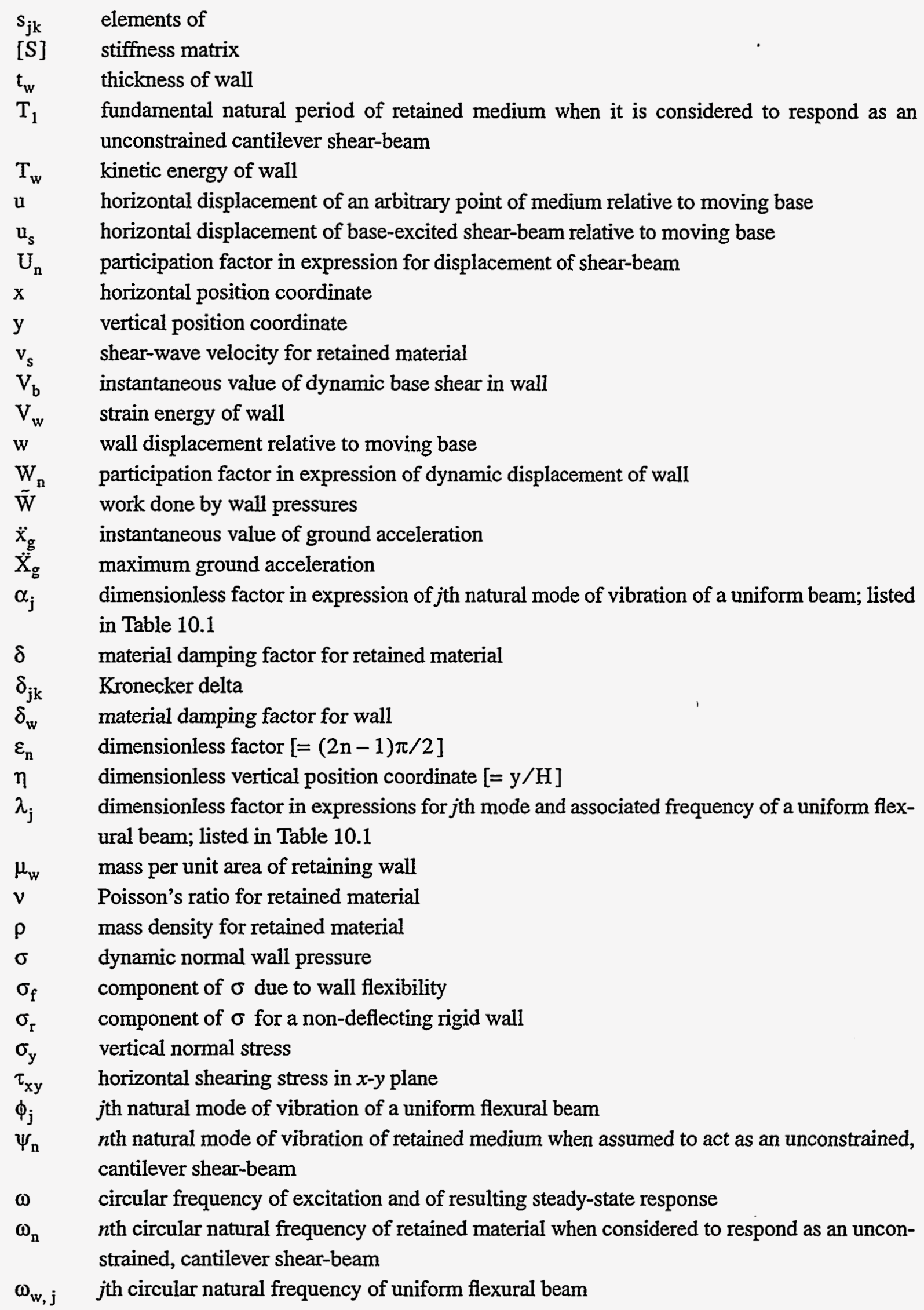

